Supplementary Information for:

\title{
Molecule Isomerism Modulates the Diradical Properties of Stable Singlet Diradicaloids
}

Joshua E. Barker, ${ }^{\dagger}$ Justin J. Dressler, ${ }^{\dagger}$ Abel Cárdenas Valdivia, ${ }^{\star}$ Ryohei Kishi, ${ }^{\S}$ Eric T. Strand, ${ }^{\dagger}$ Lev N. Zakharov, ${ }^{11}$ Samantha N. MacMillan, ${ }^{\complement}$ Carlos J. Gómez-García, ${ }^{\perp}$ Masayoshi Nakano, $*, \S, \infty, \&, \#$

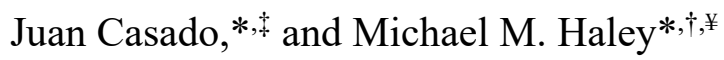

${ }^{\dagger}$ Department of Chemistry \& Biochemistry and the Materials Science Institute, University of Oregon, Eugene, Oregon 97403-1253, United States

${ }^{\ddagger}$ Department of Physical Chemistry, University of Malaga, Campus de Teatinos s/n 29071 Malaga, Spain

${ }^{\S}$ Department of Materials Engineering Science, Graduate School of Engineering Science, Osaka

University, Toyonaka, Osaka 560-8531, Japan

${ }^{11}$ CAMCOR, University of Oregon, Eugene, Oregon 97403-1433, United States

"Department of Chemistry \& Chemical Biology, Cornell University, Ithaca, New York 14853, United States

${ }^{\perp}$ Department of Inorganic Chemistry and Instituto de Ciencia Molecular, Universidad de Valencia, 46980 Paterna, Spain

${ }^{\infty}$ Center for Spintronics Research Network, Graduate School of Engineering Science, Osaka University, Toyonaka, Osaka 560-8531, Japan

${ }^{\&}$ Quantum Information and Quantum Biology Division, Institute for Open and Transdisciplinary Research Initiatives, Osaka University, Toyonaka, Osaka 560-8531, Japan

\#Institute for Molecular Science, 38 Nishigo-Naka, Myodaiji, Okazaki 444-8585, Japan

${ }^{¥}$ Phil and Penny Knight Campus for Accelerating Scientific Impact, University of Oregon, Eugene, Oregon 97403-6231, United State

Table of Contents Page

Indenofluorenes Figure S2

Computational Details S3

Cartesian Coordinates of Optimized Geometries

Experimental Details

Copies of NMR Spectra

References

S26 


\section{Indenofluorenes Figure}
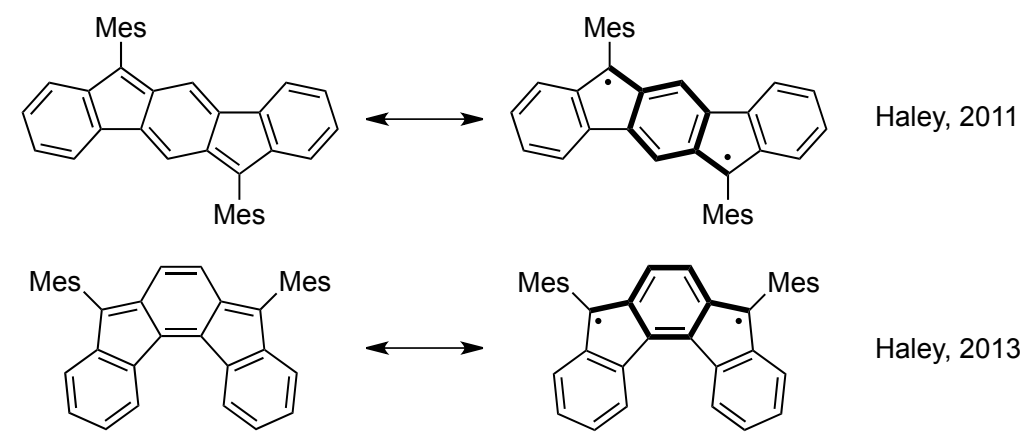

Haley, 2013

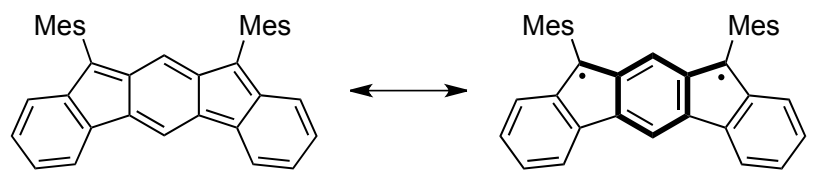

Tobe, 2013
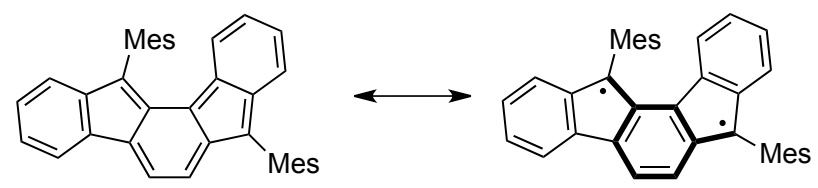

Haley, 2017

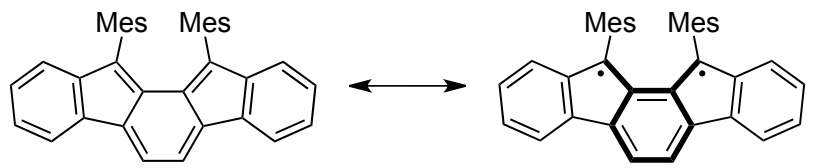

Tobe, 2011

Figure S1. Quinoidal and diradical resonance forms of the five regioisomeric indenofluorenes. 


\section{Computational Details}

Geometry optimization and frequency analysis for the singlet and triplet states were performed at the RB3LYP and UB3LYP levels, respectively, using the 6-311G* basis set. Vertical and adiabatic $\Delta E_{\mathrm{ST}}$ values were evaluated at the spin-flip non-collinear (SF-NC-)TDDFT PBE5050/6-311G* level, ${ }^{1,2}$ where zero-point vibrational energy (ZPVE) corrections for the singlet and triplet states were estimated from the results of the frequency analysis calculations at the RB3LYP and UB3LYP levels, respectively. Excitation energies were evaluated at the TDUB3LYP/6-311G* level. CASCI $(2,2)$ calculations for the estimations of VCI parameters were performed using the molecular orbitals obtained at the tuned-LC-RBLYP/6-311G* level, where an optimal range-separating parameter $\mu$ for each system was determined by IP-tuning scheme for $N$-electron system. ${ }^{3}$ These calculations were performed using Gaussian 09 (geometry optimization and excitation energy), ${ }^{4}$ GAMESS-US (CASCI), ${ }^{5}$ and Q-Chem $4.2\left(\Delta E_{\mathrm{ST}}\right)^{6}$ program packages. Diradical characters $y$ were evaluated at the PUHF/6-311 $\mathrm{G}^{*}$ [denoted as $\left.y(\mathrm{PUHF})\right]$ and tuned-LCRBLYP-CASCI $(2,2) / 6-311 \mathrm{G}^{*}$ [denoted as $y$ (CASCI)] levels. ${ }^{7}$

Table S1. Summary of calculation results for $1 \mathrm{a}$ and $1 \mathrm{~s}$.

\begin{tabular}{lcc}
\hline & 1a & 2a \\
\hline$y$ (PUHF) $[-]$ & 0.613 & 0.658 \\
\hline Vertical $\Delta E_{\mathrm{ST}}\left[\mathrm{kcal} \mathrm{mol}^{-1}\right]$ & -11.65 & -11.09 \\
Adiabatic $\Delta E_{\mathrm{ST}}\left[\mathrm{kcal} \mathrm{mol}^{-1}\right]$ & -9.37 & -8.84 \\
Adiabatic $\Delta E_{\mathrm{ST}}\left(+\mathrm{ZPVE}^{-1}\left[\mathrm{kcal} \mathrm{mol}^{-1}\right]\right.$ & -8.77 & -8.06 \\
\hline & & \\
\hline Tuned value of $\mu\left[\mathrm{bohr}^{-1}\right]$ & 0.1444 & 0.1434 \\
$y(\mathrm{CASCI})[-]$ & 0.203 & 0.210 \\
Vertical $\Delta E_{\mathrm{ST}}(\mathrm{CASCI})\left[\mathrm{kcal} \mathrm{mol}^{-1}\right]$ & -19.8 & -17.5 \\
$\left|t_{a b}\right|[\mathrm{eV}]$ & 1.031 & 0.905 \\
$f_{\mathrm{ST}}(y)[-]$ & -0.655 & -0.632 \\
$U / 2=K_{g u}^{\mathrm{M}}[\mathrm{eV}]$ & 1.563 & 1.404 \\
$(U / 2) f_{\mathrm{ST}}(y)[\mathrm{eV}]$ & -1.025 & -0.887 \\
$J_{g g}^{\mathrm{M}}[\mathrm{eV}]$ & 4.417 & 4.225 \\
$J_{u u}^{\mathrm{M}}[\mathrm{eV}]$ & 4.755 & 4.394 \\
$J_{g u}^{\mathrm{M}}[\mathrm{eV}]$ & 4.420 & 4.179 \\
$2 K_{a b}[\mathrm{eV}]$ & 0.165 & 0.130 \\
\hline
\end{tabular}


HONO
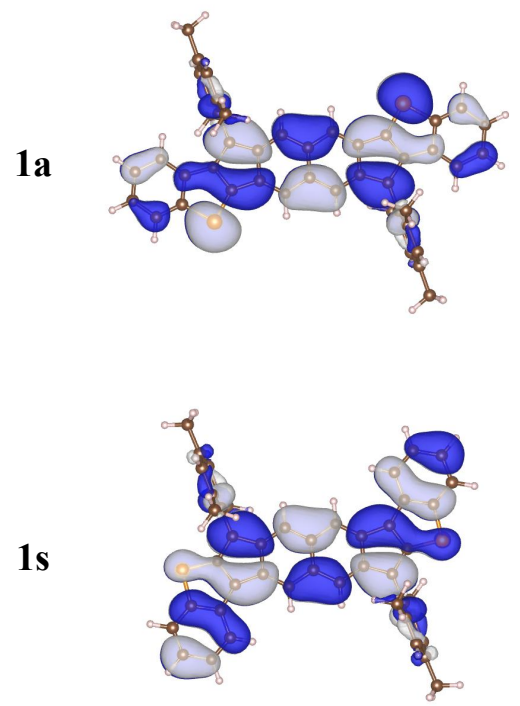

LUNO
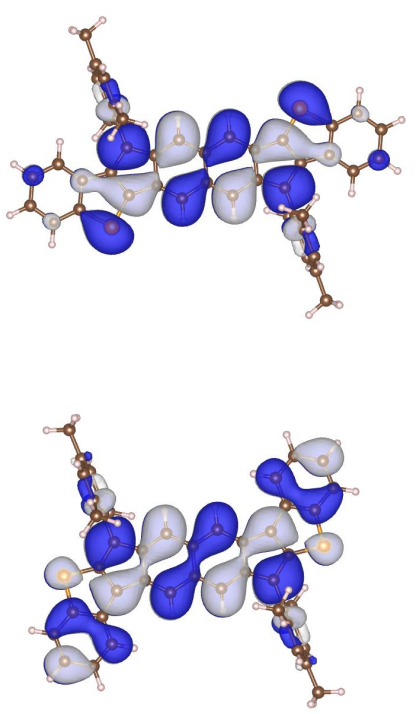

Odd-electron density
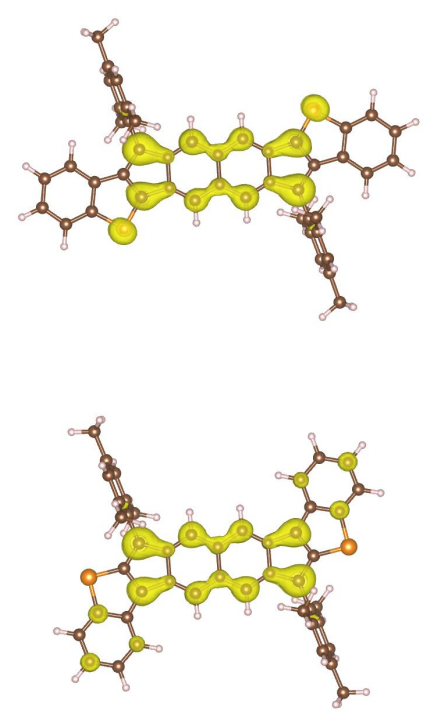

Figure S2. Spatial distributions of natural orbitals (NOs) and odd-electron density calculated at the tunedLC-RBLYP-CASCI $(2,2) / 6-311 \mathrm{G}^{*}$ level. White and blue meshes for NO maps represent the isosurfaces with the contour values of \pm 0.01 a.u. Yellow mesh for odd-electron density maps represents the isosurface with the contour value of 0.0005 a.u. 
$1 \mathbf{a}$

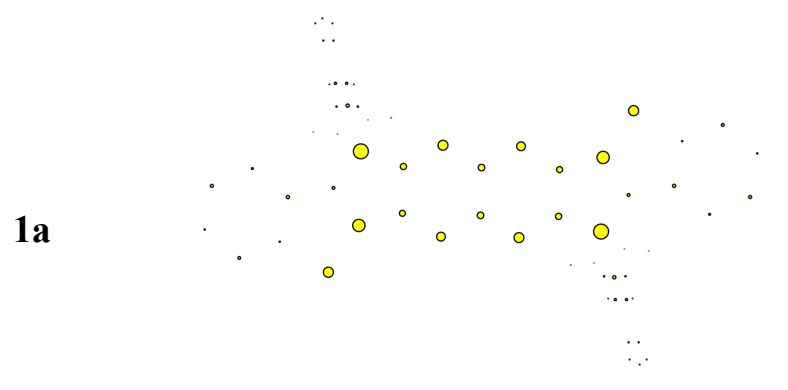

$\therefore$

$$
y=0.203
$$
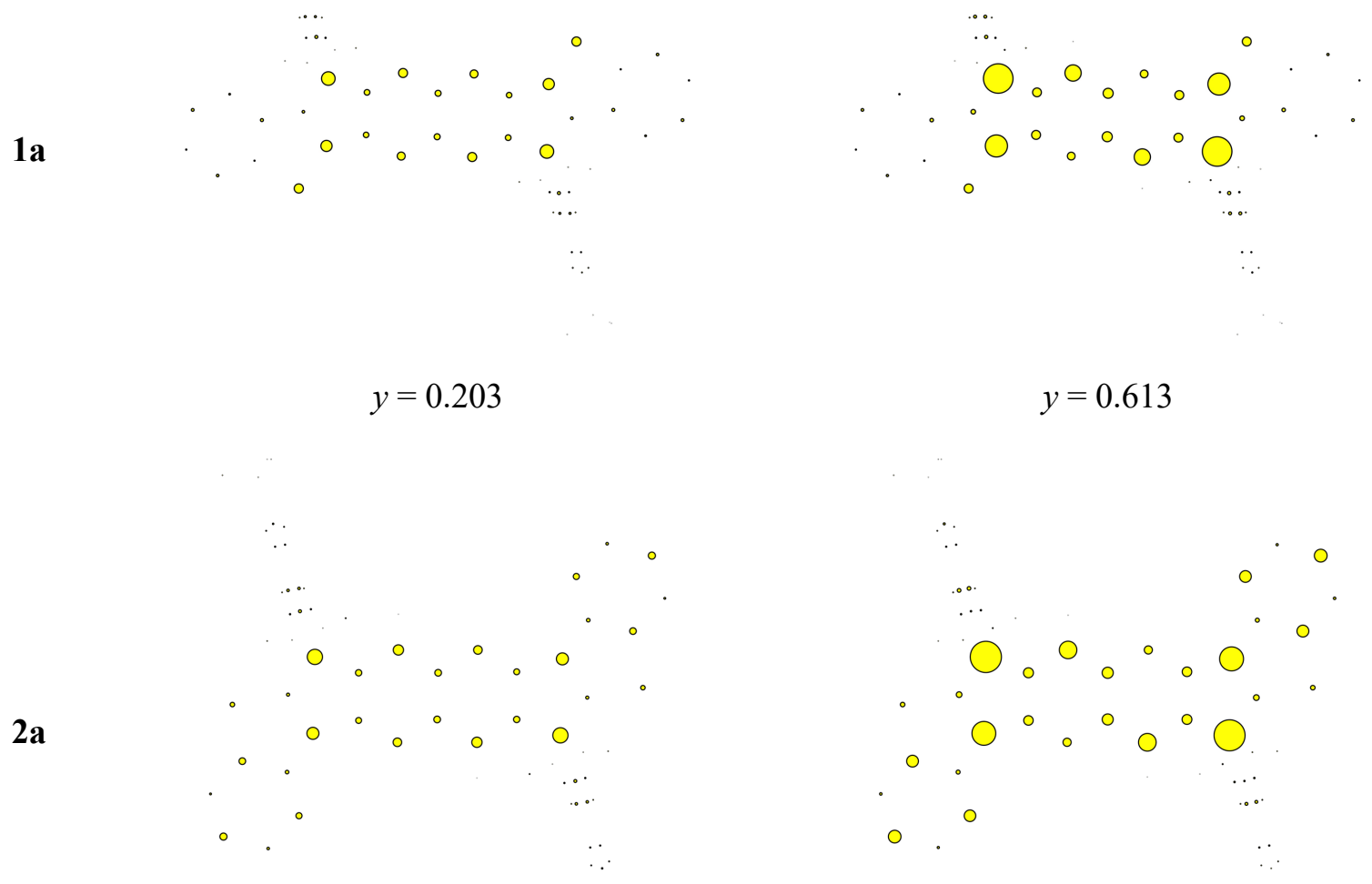

$$
y=0.210
$$

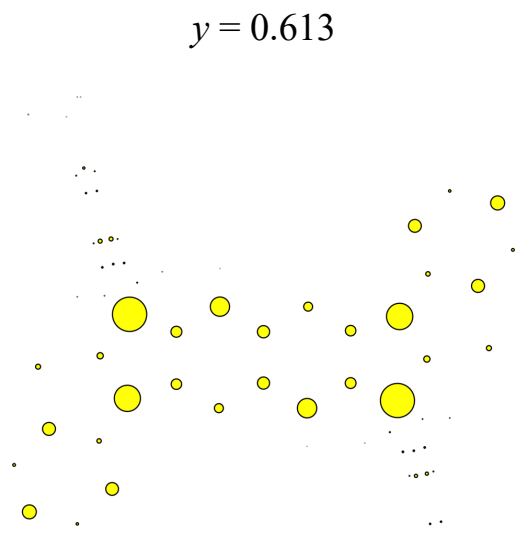

Figure S3. Mulliken population analysis for the odd-electron density calculated at the tuned-LC-RBLYPCASCI $(2,2) / 6-311 G^{*}$ and PUHF/6-311G* levels. Note that the sum of odd-electron populations (oddelectron number) corresponds to $2 y$ at the given level of approximation. ${ }^{7}$ 
Table S2. Excitation energies of 1a calculated at the TD-UB3LYP/6-311G* level.

\begin{tabular}{|c|c|c|c|c|}
\hline $\begin{array}{c}\text { Excited } \\
\text { state }\end{array}$ & $\begin{array}{c}\text { Excitation energy [eV] } \\
\text { (wavelength }[\mathrm{nm}])\end{array}$ & Excitation amplitudes $^{a}$ & $<S^{2}>^{b}$ & $\begin{array}{l}\text { Oscillator } \\
\text { strength }\end{array}$ \\
\hline 1 & $0.752(1649)$ & $\begin{array}{l}0.724(\mathrm{H} \alpha \rightarrow \mathrm{L} \alpha) \\
0.724(\mathrm{H} \beta \rightarrow \mathrm{L} \beta) \\
0.184(\mathrm{H} \alpha \leftarrow \mathrm{L} \alpha) \\
0.184(\mathrm{H} \beta \leftarrow \mathrm{L} \beta)\end{array}$ & 1.081 & 0.0000 \\
\hline 2 & $1.414(877)$ & $\begin{array}{r}0.700(\mathrm{H}-1 \alpha \rightarrow \mathrm{L} \alpha) \\
-0.700(\mathrm{H}-1 \beta \rightarrow \mathrm{L} \beta)\end{array}$ & 1.788 & 0.0005 \\
\hline 3 & $1.508(822)$ & $\begin{array}{l}0.687(\mathrm{H}-1 \alpha \rightarrow \mathrm{L} \alpha) \\
0.687(\mathrm{H}-1 \beta \rightarrow \mathrm{L} \beta) \\
0.109(\mathrm{H}-3 \alpha \rightarrow \mathrm{L} \alpha) \\
0.109(\mathrm{H}-3 \beta \rightarrow \mathrm{L} \beta)\end{array}$ & 0.290 & 0.0000 \\
\hline 4 & $1.765(703)$ & $\begin{array}{l}0.681(\mathrm{H} \alpha \rightarrow \mathrm{L} \alpha) \\
-0.681(\mathrm{H} \beta \rightarrow \mathrm{L} \beta) \\
-0.186(\mathrm{H}-1 \alpha \rightarrow \mathrm{L}+1 \alpha) \\
0.186(\mathrm{H}-1 \beta \rightarrow \mathrm{L}+1 \beta) \\
-0.110(\mathrm{H} \alpha \leftarrow \mathrm{L} \alpha) \\
0.110(\mathrm{H} \beta \leftarrow \mathrm{L} \beta)\end{array}$ & 0.005 & 0.5447 \\
\hline 5 & $2.192(566)$ & $\begin{array}{r}-0.668(\mathrm{H} \alpha \rightarrow \mathrm{L}+1 \alpha) \\
0.668(\mathrm{H} \beta \rightarrow \mathrm{L}+1 \beta) \\
-0.172(\mathrm{H}-1 \alpha \rightarrow \mathrm{L} \alpha) \\
0.172(\mathrm{H}-1 \beta \rightarrow \mathrm{L} \beta)\end{array}$ & 1.742 & 0.0007 \\
\hline
\end{tabular}

${ }^{a} \mathrm{H}$ and $\mathrm{L}$ represent HOMO and LUMO, respectively. Excitations with amplitudes larger than 0.1 are shown. ${ }^{b}<S^{2}>$ of the ground state at the UB3LYP/6-311G* level is 0.376 . 
Table S3. Excitation energies of 2a calculated at the TD-UB3LYP/6-311G* level.

\begin{tabular}{|c|c|c|c|c|}
\hline $\begin{array}{l}\text { Excited } \\
\text { state }\end{array}$ & $\begin{array}{c}\text { Excitation energy }[\mathrm{eV}] \\
\text { (wavelength }[\mathrm{nm}])\end{array}$ & Excitation amplitudes $^{a}$ & $<S^{2}>^{b}$ & $\begin{array}{l}\text { Oscillator } \\
\text { strength }\end{array}$ \\
\hline 1 & 0.687 (1806) & $\begin{array}{l}0.726(\mathrm{H} \alpha \rightarrow \mathrm{L} \alpha) \\
0.726(\mathrm{H} \beta \rightarrow \mathrm{L} \beta) \\
0.190(\mathrm{H} \alpha \leftarrow \mathrm{L} \alpha) \\
0.190(\mathrm{H} \beta \leftarrow \mathrm{L} \beta)\end{array}$ & 1.118 & 0.0000 \\
\hline 2 & $1.165(1064)$ & $\begin{array}{r}-0.701(\mathrm{H}-1 \alpha \rightarrow \mathrm{L} \alpha) \\
0.701(\mathrm{H}-1 \beta \rightarrow \mathrm{L} \beta)\end{array}$ & 1.801 & 0.0022 \\
\hline 3 & $1.326(935)$ & $\begin{array}{l}0.690(\mathrm{H}-1 \alpha \rightarrow \mathrm{L} \alpha) \\
0.690(\mathrm{H}-1 \beta \rightarrow \mathrm{L} \beta) \\
0.111(\mathrm{H}-2 \alpha \rightarrow \mathrm{L} \alpha) \\
0.111(\mathrm{H}-2 \beta \rightarrow \mathrm{L} \beta)\end{array}$ & 0.289 & 0.0000 \\
\hline 4 & $1.673(741)$ & $\begin{array}{l}-0.695(\mathrm{H} \alpha \rightarrow \mathrm{L} \alpha) \\
0.695(\mathrm{H} \beta \rightarrow \mathrm{L} \beta) \\
-0.156(\mathrm{H}-1 \alpha \rightarrow \mathrm{L}+1 \alpha) \\
0.156(\mathrm{H}-1 \beta \rightarrow \mathrm{L}+1 \beta) \\
0.121(\mathrm{H} \alpha \leftarrow \mathrm{L} \alpha) \\
-0.121(\mathrm{H} \beta \leftarrow \mathrm{L} \beta)\end{array}$ & -0.029 & 0.6684 \\
\hline 5 & $2.192(566)$ & $\begin{array}{r}0.667(\mathrm{H} \alpha \rightarrow \mathrm{L}+1 \alpha) \\
0.667(\mathrm{H} \beta \rightarrow \mathrm{L}+1 \beta) \\
-0.120(\mathrm{H}-1 \alpha \rightarrow \mathrm{L} \alpha) \\
-0.120(\mathrm{H}-1 \beta \rightarrow \mathrm{L} \beta) \\
-0.105(\mathrm{H}-9 \alpha \rightarrow \mathrm{L} \alpha) \\
-0.105(\mathrm{H}-9 \beta \rightarrow \mathrm{L} \beta)\end{array}$ & 1.739 & 0.0000 \\
\hline
\end{tabular}

${ }^{a} \mathrm{H}$ and $\mathrm{L}$ represent $\mathrm{HOMO}$ and LUMO, respectively. Excitations with amplitudes larger than 0.1 are shown. ${ }^{b}<S^{2}>$ of the ground state at the UB3LYP/6-311G* level is 0.363 . 
$1 a$

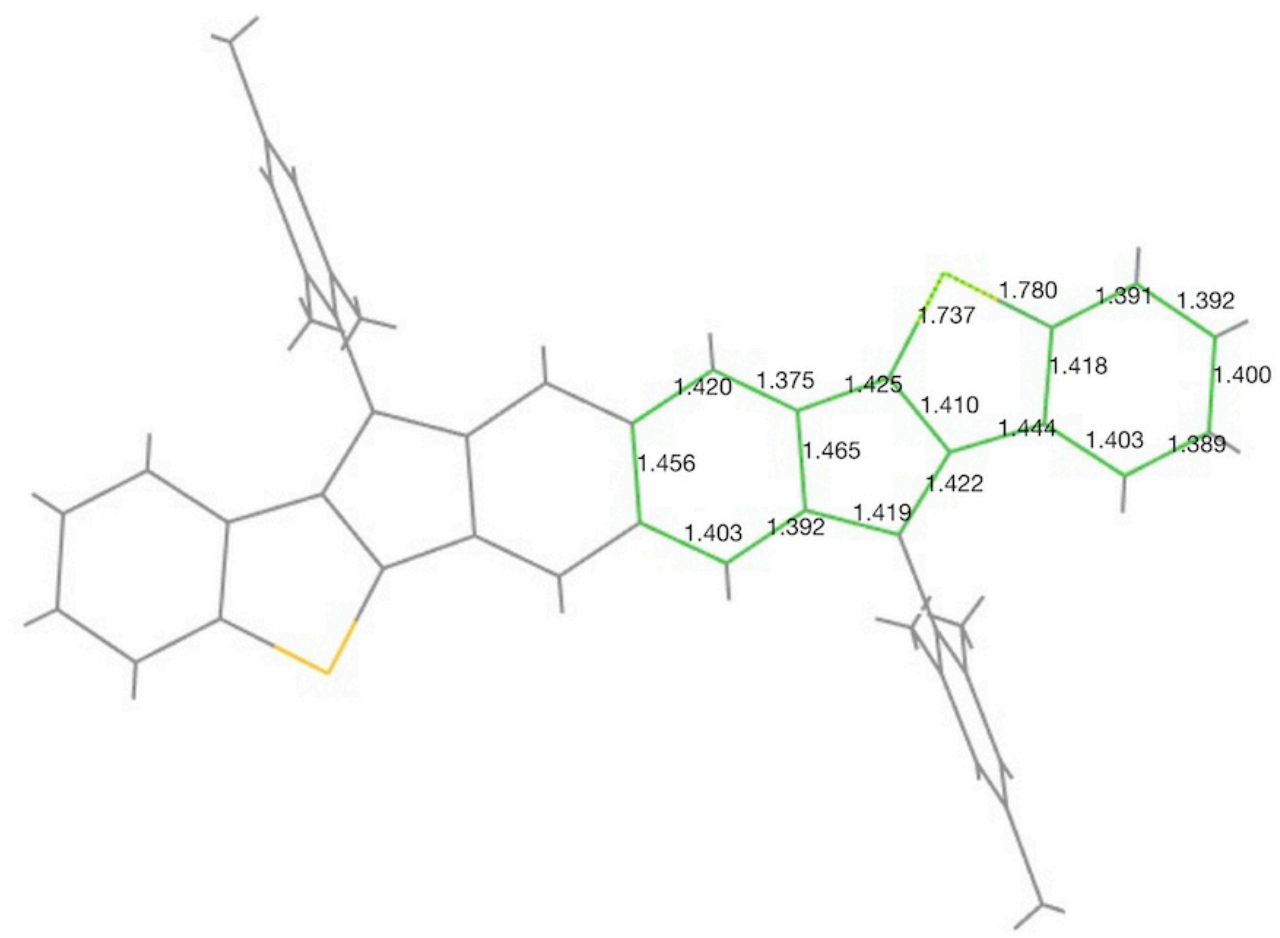

2a

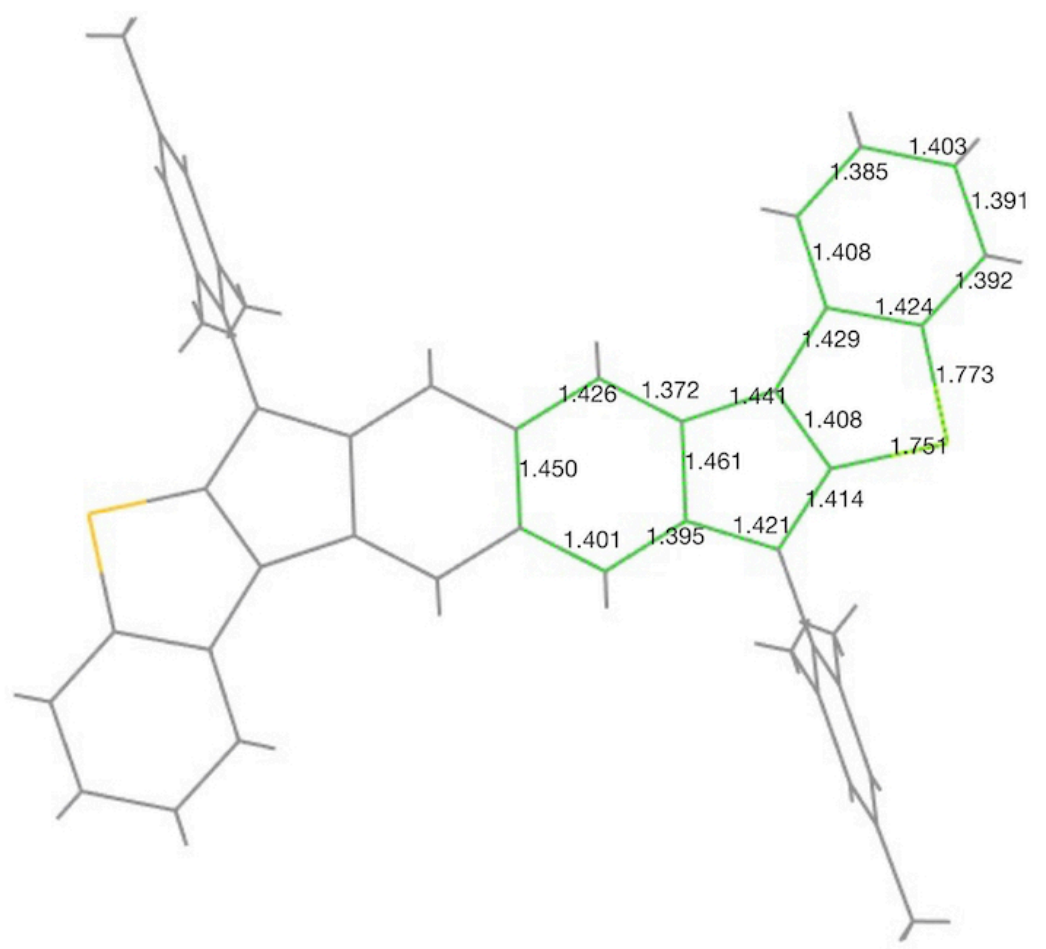

Figure S4. Selected bond lengths $(\AA)$ for the singlet state calculated at the RB3LYP/6-311G* level. 


\section{Cartesian Coordinates of Optimized Geometries}

Table S4. Optimized geometry for singlet 1a at the RB3LYP/6-311G* level (Total energy: 2570.18389983 Hartree, lowest frequency: $16.0370 \mathrm{~cm}^{-1}$ ).

\begin{tabular}{|c|c|c|c|c|c|c|c|}
\hline & $\mathrm{X}$ & $\mathrm{Y}$ & Z & & $\mathrm{X}$ & $\mathrm{Y}$ & $\mathrm{Z}$ \\
\hline $\bar{C}$ & 1.513760 & 1.100368 & 0.081290 & $\mathrm{H}$ & -0.612356 & 2.724543 & 0.016981 \\
\hline $\mathrm{C}$ & 0.167989 & 0.707925 & 0.017368 & $\mathrm{C}$ & -4.693523 & -1.571907 & -0.232430 \\
\hline $\mathrm{C}$ & 2.532057 & 0.151436 & 0.114482 & $\mathrm{C}$ & -4.897541 & -2.219356 & -1.468371 \\
\hline $\mathrm{C}$ & -0.167989 & -0.707925 & -0.017368 & $\mathrm{C}$ & -5.211173 & -2.137340 & 0.950068 \\
\hline $\mathrm{C}$ & 2.190320 & -1.273070 & 0.074742 & $\mathrm{C}$ & -5.609431 & -3.419748 & -1.495402 \\
\hline $\mathrm{C}$ & 0.875545 & -1.670148 & 0.012150 & $\mathrm{C}$ & -5.916208 & -3.340660 & 0.875197 \\
\hline $\mathrm{H}$ & 0.612356 & -2.724543 & -0.016981 & $\mathrm{H}$ & -5.768712 & -3.912626 & -2.451400 \\
\hline $\mathrm{H}$ & 1.758645 & 2.159206 & 0.105069 & $\mathrm{H}$ & -6.311972 & -3.773396 & 1.790635 \\
\hline C & -3.438018 & 1.959391 & -0.113967 & $\mathrm{C}$ & -6.124861 & -4.000250 & -0.335826 \\
\hline $\mathrm{C}$ & -3.942210 & -0.292372 & -0.177323 & $\mathrm{C}$ & 4.693523 & 1.571907 & 0.232430 \\
\hline $\mathrm{C}$ & 3.942210 & 0.292372 & 0.177323 & $\mathrm{C}$ & 4.897541 & 2.219356 & 1.468371 \\
\hline C & 3.438018 & -1.959391 & 0.113967 & $\mathrm{C}$ & 5.211173 & 2.137340 & -0.950068 \\
\hline $\mathrm{C}$ & 4.488416 & -1.020781 & 0.175192 & $\mathrm{C}$ & 5.609431 & 3.419748 & 1.495402 \\
\hline $\mathrm{C}$ & -4.488416 & 1.020781 & -0.175192 & $\mathrm{C}$ & 5.916208 & 3.340660 & -0.875197 \\
\hline C & 7.070012 & -1.075795 & 0.295234 & $\mathrm{H}$ & 5.768712 & 3.912626 & 2.451400 \\
\hline $\mathrm{C}$ & 6.797949 & -3.882831 & 0.228789 & $\mathrm{H}$ & 6.311972 & 3.773396 & -1.790635 \\
\hline $\mathrm{C}$ & -6.797949 & 3.882831 & -0.228789 & $\mathrm{C}$ & 6.124861 & 4.000250 & 0.335826 \\
\hline $\mathrm{C}$ & -7.070012 & 1.075795 & -0.295234 & $\mathrm{C}$ & -4.378168 & -1.621714 & -2.755227 \\
\hline $\mathrm{C}$ & 8.188671 & -1.898524 & 0.331260 & $\mathrm{H}$ & -3.289361 & -1.522069 & -2.747392 \\
\hline $\mathrm{C}$ & 8.056259 & -3.292212 & 0.297724 & $\mathrm{H}$ & -4.651423 & -2.240432 & -3.612299 \\
\hline $\mathrm{C}$ & -8.056259 & 3.292212 & -0.297724 & $\mathrm{H}$ & -4.782064 & -0.619079 & -2.923287 \\
\hline $\mathrm{C}$ & -8.188671 & 1.898524 & -0.331260 & $\mathrm{C}$ & -5.017499 & -1.458330 & 2.285641 \\
\hline $\mathrm{H}$ & 7.179371 & 0.002574 & 0.323110 & $\mathrm{H}$ & -5.519570 & -0.486800 & 2.320031 \\
\hline $\mathrm{H}$ & 9.177701 & -1.455643 & 0.386543 & $\mathrm{H}$ & -5.418570 & -2.068480 & 3.097361 \\
\hline $\mathrm{H}$ & 8.940401 & -3.920463 & 0.326401 & $\mathrm{H}$ & -3.960539 & -1.272318 & 2.494456 \\
\hline $\mathrm{H}$ & 6.695496 & -4.962668 & 0.204108 & $\mathrm{C}$ & -6.862203 & -5.316357 & -0.390154 \\
\hline $\mathrm{H}$ & -7.179371 & -0.002574 & -0.323110 & $\mathrm{H}$ & -7.452342 & -5.409582 & -1.305424 \\
\hline $\mathrm{H}$ & -9.177701 & 1.455643 & -0.386543 & $\mathrm{H}$ & -6.165309 & -6.161616 & -0.368678 \\
\hline $\mathrm{H}$ & -8.940401 & 3.920463 & -0.326401 & $\mathrm{H}$ & -7.539842 & -5.433648 & 0.458689 \\
\hline $\mathrm{H}$ & -6.695496 & 4.962668 & -0.204108 & $\mathrm{C}$ & 5.017499 & 1.458330 & -2.285641 \\
\hline S & 3.987989 & -3.607156 & 0.109552 & $\mathrm{H}$ & 5.519570 & 0.486800 & -2.320031 \\
\hline $\mathrm{C}$ & 5.789373 & -1.644430 & 0.224300 & $\mathrm{H}$ & 3.960539 & 1.272318 & -2.494456 \\
\hline $\mathrm{C}$ & -5.678400 & 3.057268 & -0.193311 & $\mathrm{H}$ & 5.418570 & 2.068480 & -3.097361 \\
\hline $\mathrm{C}$ & -5.789373 & 1.644430 & -0.224300 & $\mathrm{C}$ & 4.378168 & 1.621714 & 2.755227 \\
\hline $\mathrm{C}$ & 5.678400 & -3.057268 & 0.193311 & $\mathrm{H}$ & 3.289361 & 1.522069 & 2.747392 \\
\hline S & -3.987989 & 3.607156 & -0.109552 & $\mathrm{H}$ & 4.782064 & 0.619079 & 2.923287 \\
\hline $\mathrm{C}$ & -1.513760 & -1.100368 & -0.081290 & $\mathrm{H}$ & 4.651423 & 2.240432 & 3.612299 \\
\hline $\mathrm{C}$ & -0.875545 & 1.670148 & -0.012150 & $\mathrm{C}$ & 6.862203 & 5.316357 & 0.390154 \\
\hline $\mathrm{C}$ & -2.190320 & 1.273070 & -0.074742 & $\mathrm{H}$ & 7.452342 & 5.409582 & 1.305424 \\
\hline $\mathrm{C}$ & -2.532057 & -0.151436 & -0.114482 & $\mathrm{H}$ & 7.539842 & 5.433648 & -0.458689 \\
\hline $\mathrm{H}$ & -1.758645 & -2.159206 & -0.105069 & $\mathrm{H}$ & 6.165309 & 6.161616 & 0.368678 \\
\hline
\end{tabular}


Table S5. Optimized geometry for triplet 1a at the UB3LYP/6-311G* level (Total energy: -2723.86336623 Hartree, lowest frequency: $8.6876 \mathrm{~cm}^{-1}$ ).

\begin{tabular}{|c|c|c|c|c|c|c|c|}
\hline & $\mathrm{X}$ & $\mathrm{Y}$ & $\mathrm{Z}$ & & $\mathrm{X}$ & $\mathrm{Y}$ & $\mathrm{Z}$ \\
\hline S & 4.258406 & -3.268707 & -0.179031 & $\mathrm{~S}$ & -4.258406 & 3.268707 & 0.179031 \\
\hline $\mathrm{C}$ & 0.106184 & 0.710650 & -0.041536 & $\mathrm{C}$ & -0.106184 & -0.710650 & 0.041536 \\
\hline $\mathrm{C}$ & 1.430519 & 1.215549 & -0.165365 & $\mathrm{C}$ & -1.430519 & -1.215549 & 0.165365 \\
\hline $\mathrm{H}$ & 1.583899 & 2.288927 & -0.233009 & $\mathrm{H}$ & -1.583899 & -2.288927 & 0.233009 \\
\hline $\mathrm{C}$ & 2.503419 & 0.353490 & -0.200669 & $\mathrm{C}$ & -2.503419 & -0.353490 & 0.200669 \\
\hline $\mathrm{C}$ & 3.933109 & 0.607814 & -0.326959 & $\mathrm{C}$ & -3.933109 & -0.607814 & 0.326959 \\
\hline $\mathrm{C}$ & 4.568125 & -0.655073 & -0.328048 & $\mathrm{C}$ & -4.568125 & 0.655073 & 0.328048 \\
\hline $\mathrm{C}$ & 5.910295 & -1.186610 & -0.400034 & $\mathrm{C}$ & -5.910295 & 1.186610 & 0.400034 \\
\hline $\mathrm{C}$ & 7.143476 & -0.529153 & -0.514986 & $\mathrm{C}$ & -7.143476 & 0.529153 & 0.514986 \\
\hline $\mathrm{H}$ & 7.173509 & 0.553759 & -0.560305 & $\mathrm{H}$ & -7.173509 & -0.553759 & 0.560305 \\
\hline $\mathrm{C}$ & 8.317803 & -1.270381 & -0.564692 & $\mathrm{C}$ & -8.317803 & 1.270381 & 0.564692 \\
\hline $\mathrm{H}$ & 9.270615 & -0.758853 & -0.653201 & $\mathrm{H}$ & -9.270615 & 0.758853 & 0.653201 \\
\hline $\mathrm{C}$ & 8.287247 & -2.668611 & -0.500872 & $\mathrm{C}$ & -8.287247 & 2.668611 & 0.500872 \\
\hline $\mathrm{H}$ & 9.213413 & -3.232300 & -0.541297 & $\mathrm{H}$ & -9.213413 & 3.232300 & 0.541297 \\
\hline $\mathrm{C}$ & 7.076924 & -3.346316 & -0.384261 & $\mathrm{C}$ & -7.076924 & 3.346316 & 0.384261 \\
\hline $\mathrm{H}$ & 7.053272 & -4.429758 & -0.332753 & $\mathrm{H}$ & -7.053272 & 4.429758 & 0.332753 \\
\hline $\mathrm{C}$ & 5.902731 & -2.602000 & -0.334184 & $\mathrm{C}$ & -5.902731 & 2.602000 & 0.334184 \\
\hline $\mathrm{C}$ & 3.594704 & -1.667814 & -0.204373 & $\mathrm{C}$ & -3.594704 & 1.667814 & 0.204373 \\
\hline $\mathrm{C}$ & 2.283532 & -1.077272 & -0.122568 & $\mathrm{C}$ & -2.283532 & 1.077272 & 0.122568 \\
\hline $\mathrm{C}$ & 1.012838 & -1.587441 & -0.004115 & $\mathrm{C}$ & -1.012838 & 1.587441 & 0.004115 \\
\hline $\mathrm{H}$ & 0.842242 & -2.658805 & 0.053975 & $\mathrm{H}$ & -0.842242 & 2.658805 & -0.053975 \\
\hline $\mathrm{C}$ & 4.566682 & 1.942867 & -0.438011 & $\mathrm{C}$ & -4.566682 & -1.942867 & 0.438011 \\
\hline $\mathrm{C}$ & 4.978916 & 2.427348 & -1.694782 & $\mathrm{C}$ & -4.978916 & -2.427348 & 1.694782 \\
\hline $\mathrm{C}$ & 5.572260 & 3.690315 & -1.775112 & $\mathrm{C}$ & -5.572260 & -3.690315 & 1.775112 \\
\hline $\mathrm{H}$ & 5.883408 & 4.061421 & -2.748302 & $\mathrm{H}$ & -5.883408 & -4.061421 & 2.748302 \\
\hline $\mathrm{C}$ & 5.769566 & 4.486278 & -0.648381 & $\mathrm{C}$ & -5.769566 & -4.486278 & 0.648381 \\
\hline $\mathrm{C}$ & 5.352443 & 3.987949 & 0.587907 & $\mathrm{C}$ & -5.352443 & -3.987949 & -0.587907 \\
\hline $\mathrm{H}$ & 5.500669 & 4.591459 & 1.480326 & $\mathrm{H}$ & -5.500669 & -4.591459 & -1.480326 \\
\hline $\mathrm{C}$ & 4.754485 & 2.734695 & 0.715771 & $\mathrm{C}$ & -4.754485 & -2.734695 & -0.715771 \\
\hline $\mathrm{C}$ & 4.782815 & 1.606812 & -2.948125 & $\mathrm{C}$ & -4.782815 & -1.606812 & 2.948125 \\
\hline $\mathrm{H}$ & 3.749704 & 1.263279 & -3.047572 & $\mathrm{H}$ & -3.749704 & -1.263279 & 3.047572 \\
\hline $\mathrm{H}$ & 5.031584 & 2.187514 & -3.838698 & $\mathrm{H}$ & -5.031584 & -2.187514 & 3.838698 \\
\hline $\mathrm{H}$ & 5.413386 & 0.712284 & -2.949676 & $\mathrm{H}$ & -5.413386 & -0.712284 & 2.949676 \\
\hline $\mathrm{C}$ & 6.417840 & 5.845847 & -0.750357 & $\mathrm{C}$ & -6.417840 & -5.845847 & 0.750357 \\
\hline $\mathrm{H}$ & 6.619515 & 6.118336 & -1.788391 & $\mathrm{H}$ & -6.619515 & -6.118336 & 1.788391 \\
\hline $\mathrm{H}$ & 5.782126 & 6.625663 & -0.319921 & $\mathrm{H}$ & -5.782126 & -6.625663 & 0.319921 \\
\hline $\mathrm{H}$ & 7.369779 & 5.875231 & -0.210231 & $\mathrm{H}$ & -7.369779 & -5.875231 & 0.210231 \\
\hline $\mathrm{C}$ & 4.340446 & 2.231649 & 2.079024 & $\mathrm{C}$ & -4.340446 & -2.231649 & -2.079024 \\
\hline $\mathrm{H}$ & 4.809399 & 1.271122 & 2.310799 & $\mathrm{H}$ & -4.809399 & -1.271122 & -2.310799 \\
\hline $\mathrm{H}$ & 4.623154 & 2.939853 & 2.860394 & $\mathrm{H}$ & -4.623154 & -2.939853 & -2.860394 \\
\hline $\mathrm{H}$ & 3.259973 & 2.075784 & 2.144952 & $\mathrm{H}$ & -3.259973 & -2.075784 & -2.144952 \\
\hline
\end{tabular}


Table S6. Optimized geometry for singlet 2a at the RB3LYP/6-311G* level (Total energy: -2570.19601268 Hartree, lowest frequency: $14.3984 \mathrm{~cm}^{-1}$ ).

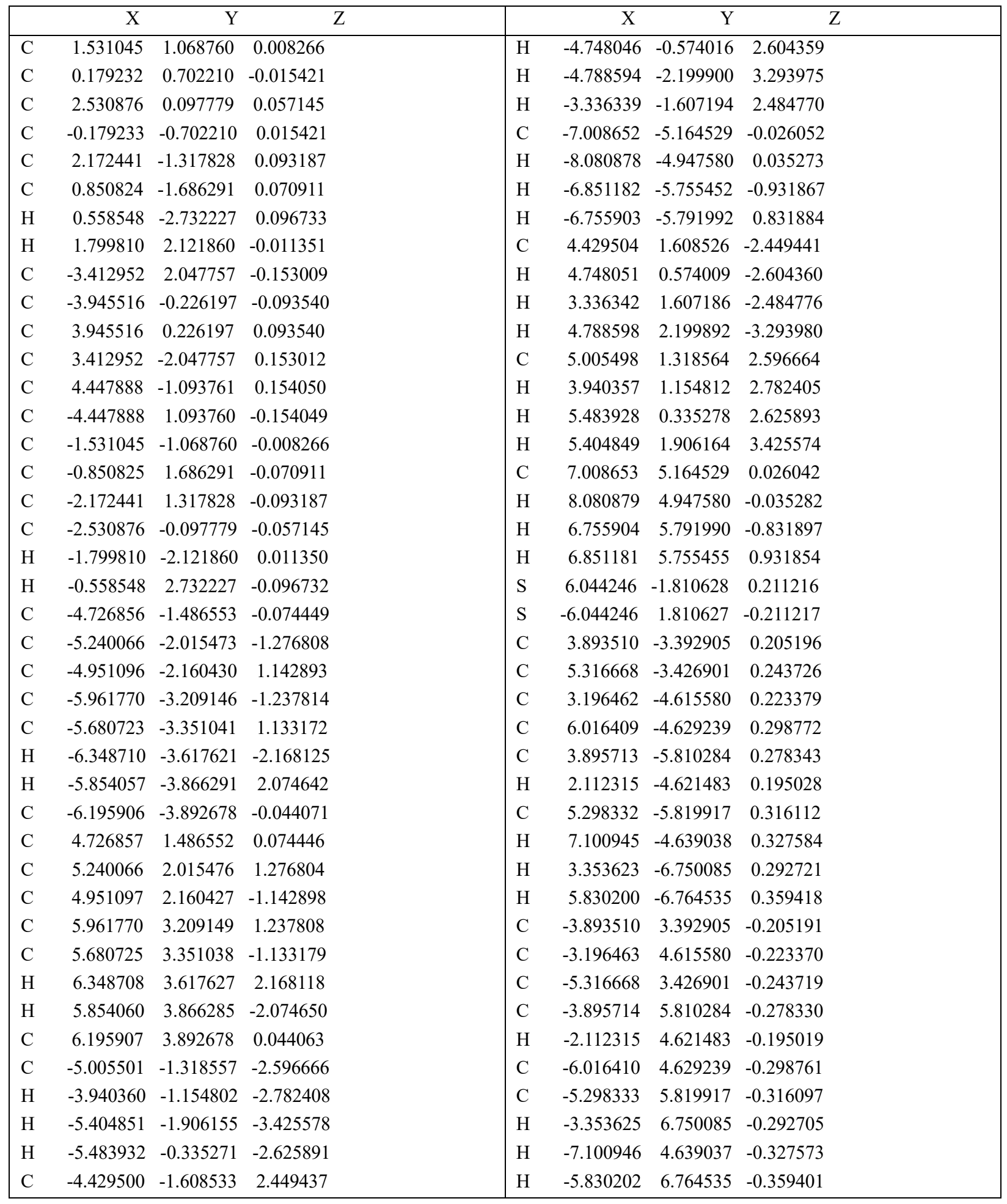


Table S7. Optimized geometry for triplet 2a at the UB3LYP/6-311G* level (Total energy: -2570.18606481 Hartree, lowest frequency: $14.5734 \mathrm{~cm}^{-1}$ ).

\begin{tabular}{|c|c|c|c|c|c|c|c|c|}
\hline & $\mathrm{X}$ & $\mathrm{Y}$ & $\mathrm{Z}$ & & $\mathrm{X}$ & $\mathrm{Y}$ & $\mathrm{Z}$ & \\
\hline $\mathrm{C}$ & 1.550284 & 1.066269 & 0.004515 & $\mathrm{H}$ & -4.834923 & -0.611353 & 2.635790 & \\
\hline $\mathrm{C}$ & 0.176380 & 0.694965 & -0.017089 & $\mathrm{H}$ & -4.726557 & -2.250668 & 3.283976 & \\
\hline $\mathrm{C}$ & 2.527893 & 0.099981 & 0.052986 & $\mathrm{H}$ & -3.351555 & -1.527982 & 2.444106 & \\
\hline $\mathrm{C}$ & -0.176380 & -0.694965 & 0.017090 & $\mathrm{C}$ & -6.997416 & -5.190412 & -0.028710 & \\
\hline $\mathrm{C}$ & 2.173017 & -1.301661 & 0.092006 & $\mathrm{H}$ & -8.070735 & -4.985784 & 0.052939 & \\
\hline $\mathrm{C}$ & 0.852282 & -1.678855 & 0.073674 & $\mathrm{H}$ & -6.848844 & -5.769870 & -0.943354 & \\
\hline $\mathrm{H}$ & 0.564195 & -2.725130 & 0.103853 & $\mathrm{H}$ & -6.723729 & -5.824215 & 0.818112 & \\
\hline $\mathrm{H}$ & 1.815796 & 2.119552 & -0.016693 & $\mathrm{C}$ & 4.441741 & 1.613990 & -2.444091 & \\
\hline $\mathrm{C}$ & -3.424852 & 2.038418 & -0.155118 & $\mathrm{H}$ & 4.834916 & 0.611353 & -2.635789 & \\
\hline $\mathrm{C}$ & -3.985621 & -0.223480 & -0.092247 & $\mathrm{H}$ & 3.351552 & 1.527988 & -2.444106 & \\
\hline $\mathrm{C}$ & 3.985621 & 0.223479 & 0.092247 & $\mathrm{H}$ & 4.726558 & 2.250667 & -3.283975 & \\
\hline $\mathrm{C}$ & 3.424852 & -2.038418 & 0.155118 & $\mathrm{C}$ & 5.051709 & 1.311184 & 2.594610 & \\
\hline $\mathrm{C}$ & 4.470422 & -1.086397 & 0.156884 & $\mathrm{H}$ & 3.990274 & 1.123271 & 2.779030 & \\
\hline $\mathrm{C}$ & -4.470422 & 1.086397 & -0.156884 & $\mathrm{H}$ & 5.552929 & 0.339252 & 2.622580 & \\
\hline $\mathrm{C}$ & -1.550284 & -1.066269 & -0.004514 & $\mathrm{H}$ & 5.436286 & 1.906026 & 3.425367 & \\
\hline $\mathrm{C}$ & -0.852282 & 1.678855 & -0.073673 & $\mathrm{C}$ & 6.997416 & 5.190412 & 0.028710 & \\
\hline $\mathrm{C}$ & -2.173017 & 1.301661 & -0.092006 & $\mathrm{H}$ & 8.070736 & 4.985783 & -0.052937 & \\
\hline $\mathrm{C}$ & -2.527893 & -0.099981 & -0.052986 & $\mathrm{H}$ & 6.723731 & 5.824214 & -0.818113 & \\
\hline $\mathrm{H}$ & -1.815796 & -2.119552 & 0.016694 & $\mathrm{H}$ & 6.848844 & 5.769870 & 0.943353 & \\
\hline $\mathrm{H}$ & -0.564195 & 2.725130 & -0.103852 & $\mathrm{~S}$ & 6.061463 & -1.822654 & 0.219378 & \\
\hline $\mathrm{C}$ & -4.756765 & -1.487566 & -0.075144 & $\mathrm{~S}$ & -6.061463 & 1.822654 & -0.219378 & \\
\hline $\mathrm{C}$ & -5.271676 & -2.016464 & -1.276585 & $\mathrm{C}$ & 3.890631 & -3.382180 & 0.209428 & \\
\hline $\mathrm{C}$ & -4.963445 & -2.171037 & 1.140213 & $\mathrm{C}$ & 5.315817 & -3.431746 & 0.251752 & \\
\hline $\mathrm{C}$ & -5.980797 & -3.217778 & -1.238295 & $\mathrm{C}$ & 3.178466 & -4.598378 & 0.226413 & \\
\hline $\mathrm{C}$ & -5.679949 & -3.369117 & 1.130085 & $\mathrm{C}$ & 6.000165 & -4.640963 & 0.309355 & \\
\hline $\mathrm{H}$ & -6.369404 & -3.626228 & -2.167917 & $\mathrm{C}$ & 3.864651 & -5.799969 & 0.283970 & \\
\hline $\mathrm{H}$ & -5.839867 & -3.891467 & 2.070031 & $\mathrm{H}$ & 2.094702 & -4.590924 & 0.194877 & \\
\hline $\mathrm{C}$ & -6.198754 & -3.909701 & -0.046378 & $\mathrm{C}$ & 5.267244 & -5.824380 & 0.325548 & \\
\hline $\mathrm{C}$ & 4.756765 & 1.487566 & 0.075144 & $\mathrm{H}$ & 7.084375 & -4.664317 & 0.340972 & \\
\hline $\mathrm{C}$ & 5.271676 & 2.016463 & 1.276585 & $\mathrm{H}$ & 3.312862 & -6.734061 & 0.297383 & \\
\hline $\mathrm{C}$ & 4.963444 & 2.171037 & -1.140212 & $\mathrm{H}$ & 5.788544 & -6.774753 & 0.370805 & \\
\hline $\mathrm{C}$ & 5.980797 & 3.217778 & 1.238296 & $\mathrm{C}$ & -3.890631 & 3.382180 & -0.209428 & \\
\hline $\mathrm{C}$ & 5.679949 & 3.369116 & -1.130085 & $\mathrm{C}$ & -3.178466 & 4.598379 & -0.226414 & \\
\hline $\mathrm{H}$ & 6.369405 & 3.626227 & 2.167917 & $\mathrm{C}$ & -5.315817 & 3.431746 & -0.251753 & \\
\hline $\mathrm{H}$ & 5.839866 & 3.891467 & -2.070031 & $\mathrm{C}$ & -3.864651 & 5.799969 & -0.283971 & \\
\hline $\mathrm{C}$ & 6.198755 & 3.909701 & 0.046378 & $\mathrm{H}$ & -2.094702 & 4.590924 & -0.194877 & \\
\hline $\mathrm{C}$ & -5.051707 & -1.311185 & -2.594610 & $\mathrm{C}$ & -6.000165 & 4.640963 & -0.309356 & \\
\hline $\mathrm{H}$ & -3.990271 & -1.123277 & -2.779031 & $\mathrm{C}$ & -5.267244 & 5.824380 & -0.325550 & \\
\hline $\mathrm{H}$ & -5.436289 & -1.906024 & -3.425366 & $\mathrm{H}$ & -3.312862 & 6.734061 & -0.297384 & \\
\hline $\mathrm{H}$ & -5.552922 & -0.339249 & -2.622578 & $\mathrm{H}$ & -7.084375 & 4.664317 & -0.340973 & \\
\hline $\mathrm{C}$ & -4.441743 & -1.613989 & 2.444092 & $\mathrm{H}$ & -5.788544 & 6.774753 & -0.370807 & \\
\hline
\end{tabular}




\section{Experimental Details}

General. All air-sensitive manipulations were carried out under an inert atmosphere using standard Schlenk technique. For moisture sensitive reactions, THF and toluene were refluxed with $\mathrm{Na}$ benzophenone ketyl for $24 \mathrm{~h}$ prior to distillation and use. Silica gel (240-300 mesh) was used for column chromatography. All other reagents were purchased and used as received. NMR spectra were recorded on a Bruker Avance III HD 500 equipped with a Prodigy multinuclear cryoprobe $\left({ }^{1} \mathrm{H}: 500 \mathrm{MHz},{ }^{2} \mathrm{D}: 77 \mathrm{MHz}\right)$ or Bruker Avance III HD 600 equipped with a Prodigy multinuclear cryoprobe $\left({ }^{1} \mathrm{H}: 600 \mathrm{MHz},{ }^{13} \mathrm{C}: 151 \mathrm{MHz}\right) \mathrm{NMR}$ spectrometer at room temperature (unless otherwise noted). ${ }^{1} \mathrm{H}$ and ${ }^{13} \mathrm{C}$ NMR chemical shifts $(\delta)$ are expressed in ppm relative to the residual non-deuterated solvent reference $\left(\mathrm{CDCl}_{3}:{ }^{1} \mathrm{H} 7.26 \mathrm{ppm},{ }^{13} \mathrm{C} 77.16 \mathrm{ppm} ; \mathrm{CD}_{2} \mathrm{Cl}_{2}:{ }^{1} \mathrm{H} 5.32 \mathrm{ppm},{ }^{13} \mathrm{C}\right.$ 53.84 ppm; DMSO- $\left.d_{6}:{ }^{1} \mathrm{H} 2.50 \mathrm{ppm},{ }^{13} \mathrm{C} 39.52 \mathrm{ppm}\right)$. UV-Vis spectra were recorded on an Agilent Technologies Cary $60 \mathrm{UV}-\mathrm{Vis}$ spectrometer in HPLC grade $\mathrm{CH}_{2} \mathrm{Cl}_{2}$. anti-Dione, ${ }^{8}$ compound $3^{9}$ and 3-(4,4,5,5-tetramethyl-1,3,2-dioxaborolan-2-yl)benzo[b]thiophene ${ }^{10}$ were prepared according to literature procedures.

Diester 4. A two-neck round-bottom flask fitted with a condenser was charged with bistriflate $3^{9}$ (0.500 g, $0.925 \mathrm{mmol}, 1$ equiv.), benzothiophene 3-boronpinacolate ester ${ }^{10}(0.601 \mathrm{~g}$, $2.31 \mathrm{mmol}, 2.5$ equiv.), $\mathrm{K}_{3} \mathrm{PO}_{4}$ (0.589 g, $2.78 \mathrm{mmol}, 3$ equiv.), $\mathrm{Pd}(\mathrm{OAc})_{2}$ (8.3 mg, $0.037 \mathrm{mmol}$, 0.04 equiv.), and SPhos (30.4 mg, $0.074 \mathrm{mmol}, 0.08$ equiv.). These solids were then placed under $\mathrm{N}_{2}$ atmosphere and dissolved in toluene $(40 \mathrm{~mL})$ and $\mathrm{H}_{2} \mathrm{O}(1 \mathrm{~mL})$ that had been sparged with $\mathrm{N}_{2}$ for $1.5 \mathrm{~h}$. After refluxing overnight and cooling to room temperature, the reaction was quenched with $\mathrm{H}_{2} \mathrm{O}$ and poured over filter paper. The precipitate was washed with $\mathrm{H}_{2} \mathrm{O}$ and $\mathrm{MeOH}$ to yield diester 4 (409 mg, 87\%) as a yellow solid. ${ }^{1} \mathrm{H}$ NMR (500 MHz, $\left.\mathrm{CDCl}_{3}, 25{ }^{\circ} \mathrm{C}\right) \delta 8.54(\mathrm{~s}, 2 \mathrm{H}), 8.09$ (s, 2H), $7.94(\mathrm{~d}, J=8.0 \mathrm{~Hz}, 2 \mathrm{H}), 7.51$ (d, $J=7.8 \mathrm{~Hz}, 2 \mathrm{H}), 7.46$ (s, 2H), 7.39 (t, $J=7.3 \mathrm{~Hz}, 2 \mathrm{H})$, $7.35(\mathrm{t}, J=7.4 \mathrm{~Hz}, 2 \mathrm{H}), 3.53(\mathrm{~s}, 6 \mathrm{H}) ;{ }^{13} \mathrm{C} \mathrm{NMR}\left(151 \mathrm{MHz}, \mathrm{CDCl}_{3}, 25{ }^{\circ} \mathrm{C}\right) \delta(\mathrm{ppm}) 168.02,139.82$, 139.21, 136.80, 133.61, 133.24, 132.05, 131.58, 131.07, 124.58, 123.93, 123.02, 122.32, 52.50; HRMS $\left(\mathrm{ES}^{+}\right)(\mathrm{m} / \mathrm{z})$, calculated for $\mathrm{C}_{30} \mathrm{H}_{20} \mathrm{O}_{4} \mathrm{NaS}_{2}(\mathrm{M}+\mathrm{Na})^{+} 531.0701$, found 531.0654 .

syn-Dione 5. A round-bottom flask fitted with a condenser was charged with diester $3(0.400$ g, 0.786 mmol, 1 equiv.), $\mathrm{KOH}$ (0.706 g, $12.6 \mathrm{mmol}, 16$ equiv.), $\mathrm{EtOH}(60 \mathrm{~mL})$, and $\mathrm{H}_{2} \mathrm{O}$ (15 mL). After refluxing the flask overnight, the reaction was cooled and the EtOH evaporated. Concentrated $\mathrm{HCl}$ was slowly added to the aqueous solution and a precipitate formed, which was isolated and washed with $\mathrm{H}_{2} \mathrm{O}$ to yield the diacid intermediate as a yellow solid that was carried on without further purification. 
To a suspension of the diacid $\left(0.345 \mathrm{~g}, 0.718 \mathrm{mmol}, 1\right.$ equiv.) in $\mathrm{CH}_{2} \mathrm{Cl}_{2}(40 \mathrm{~mL})$ was added 3 drops of DMF followed by oxalyl chloride ( $0.24 \mathrm{ml}, 2.87 \mathrm{mmol}, 4.0$ equiv.). After $12 \mathrm{~h}$, the volatiles were removed under reduced pressure. The crude acid chloride was dissolved in $\mathrm{CH}_{2} \mathrm{Cl}_{2}(40 \mathrm{~mL})$ and solid $\mathrm{AlCl}_{3}(0.479 \mathrm{~g}, 3.59 \mathrm{mmol}, 5$ equiv. $)$ was added to the flask. The reaction was stirred overnight and then poured into an $\mathrm{HCl}$-ice mixture, precipitating the dione. The solid was filtered and washed successively with $\mathrm{H}_{2} \mathrm{O}, \mathrm{CH}_{2} \mathrm{Cl}_{2}$ and acetone to afford dione $\mathbf{5}$ as a purple solid $(0.302 \mathrm{~g}, 88 \%)$ that was too insoluble to obtain NMR spectra. HRMS $\left(\mathrm{ES}^{+}\right)(\mathrm{m} / \mathrm{z})$, calculated for $\mathrm{C}_{28} \mathrm{H}_{13} \mathrm{O}_{2} \mathrm{~S}_{2}(\mathrm{M}+\mathrm{H})^{+}$445.0351, found 445.0352.

syn-IIDBT 2a. In an oven-dried round bottom flask, a suspension of dione $5(0.195 \mathrm{~g}, 0.439$ mmol, 1 equiv.) in dry THF $(20 \mathrm{~mL})$ was cooled to $-78{ }^{\circ} \mathrm{C}$ under a $\mathrm{N}_{2}$ atmosphere. In a separate oven-dried round bottom flask, 2-bromomesitylene $(0.537 \mathrm{~mL}, 3.51 \mathrm{mmol}, 8$ equiv. $)$ was dissolved in dry THF $(20 \mathrm{~mL})$, cooled to $-78^{\circ} \mathrm{C}$ under a $\mathrm{N}_{2}$ atmosphere, and $n$-BuLi (2.5 $\mathrm{M}$ in hexanes, 1.32 $\mathrm{mL}, 3.29 \mathrm{mmol}, 7.5$ equiv.) was added dropwise. After stirring the mixture at $-78{ }^{\circ} \mathrm{C}$ for $1 \mathrm{~h}$, the aryl lithiate was transferred via cannula to the flask containing the dione. This reaction mixture was stirred for $4 \mathrm{~h}$ at $-78{ }^{\circ} \mathrm{C}$, then slowly warmed to room temperature overnight with stirring. The reaction was then quenched with a saturated aq. $\mathrm{NH}_{4} \mathrm{Cl}$ solution and extracted with $\mathrm{CH}_{2} \mathrm{Cl}_{2}$ $(3 \times)$. The combined organic layer was washed with brine, dried $\left(\mathrm{MgSO}_{4}\right)$ and concentrated in vacuo. The resulting crude residue was passed through a silica plug eluting with hexanes, followed by a $\mathrm{CH}_{2} \mathrm{Cl}_{2}$ wash, to provide the desired diol that was carried onto the reductive dearomatization step without further purification.

In a single-neck round-bottom flask the crude diol $(0.140 \mathrm{~g}, 0.204 \mathrm{mmol}, 1$ equiv. $)$ and anhydrous $\mathrm{SnCl}_{2}$ (0.154 g, $0.84 \mathrm{mmol}, 4$ equiv.) were dissolved in dry degassed toluene $(60 \mathrm{~mL})$. Trifluoroacetic acid (5 drops) was added and this mixture was then vigorously stirred. The reaction was monitored via TLC $\left(9: 1\right.$ hexanes/ $\left.\mathrm{CH}_{2} \mathrm{Cl}_{2}\right)$. After $3 \mathrm{~h}$, the solvent was evaporated and the solid was washed with cold $\mathrm{MeCN}$ to remove trace impurities. The remaining solid was redissolved in $\mathrm{CHCl}_{3}$ and $\mathrm{MeCN}$ was layered over the solution to furnish syn-IIDBT 2a as a deep green solid $(0.055 \mathrm{mg}, 20 \%$ from $\mathbf{5})$. Unfortunately, 2a proved to be poorly soluble so that only its proton NMR spectra could be obtained. ${ }^{1} \mathrm{H}$ NMR $\left(500 \mathrm{MHz}, \mathrm{CDCl}_{3}, 25^{\circ} \mathrm{C}\right) \delta(\mathrm{ppm}) 7.71(\mathrm{~d}, J=7.9 \mathrm{~Hz}$, 2H), 7.55 (d, J=8.0 Hz, 2H), 7.24 (m, 2H), 7.17-7.13 (m, 4H), 6.99 (s, 4H), 6.64 (s, 2H), 2.37 (s, $6 \mathrm{H}), 2.28(\mathrm{~s}, 12 \mathrm{H})$; HRMS $\left(\mathrm{ES}^{+}\right)(\mathrm{m} / z)$, calculated for $\mathrm{C}_{46} \mathrm{H}_{35} \mathrm{~S}_{2}(\mathrm{M}+\mathrm{H})^{+} 651.2175$, found 651.2174 . 
syn-IIDBT 2b. Following the procedure described above for 2a, dione 5 (0.115 g, 0.26 mmol, 1 equiv.), 2-bromo-5-t-butyl-1,3-dimethylbenzene (0.625 g, 2.59 mmol, 10 equiv.), and $n$ BuLi (2.5 M in hexanes, $0.984 \mathrm{~mL}, 2.46 \mathrm{mmol}$, 9.5 equiv.) were reacted to give the crude diol. Dearomatization of the crude diol $(0.130 \mathrm{~g}, 0.17 \mathrm{mmol}, 1$ equiv. $)$ with anhydrous $\mathrm{SnCl}_{2}(0.125 \mathrm{~g}$, $0.68 \mathrm{mmol}, 4$ equiv.) gave a deep green solution which was reacted for $4 \mathrm{~h}$ and then filtered through a pad of celite. The filtrate was concentrated, triturated with $\mathrm{MeCN}$, and filtered to yield syn-IIDBT 2b as a deep green solid (40 mg, 22\% from 5). ${ }^{1} \mathrm{H} \mathrm{NMR}\left(600 \mathrm{MHz}, \mathrm{CD}_{2} \mathrm{Cl}_{2}, 25{ }^{\circ} \mathrm{C}\right) \delta 7.74(\mathrm{~d}, J=$ $6.8 \mathrm{~Hz}, 2 \mathrm{H}), 7.58$ (d, J=8.0 Hz, 2H), 7.30-7.26 (m, 2H), 7.23-7.16 (m, 8H), 6.67 (s, 2H), 2.31 (s, $12 \mathrm{H}), 1.38(\mathrm{~s}, 18 \mathrm{H}) ;{ }^{13} \mathrm{C} \mathrm{NMR}\left(126 \mathrm{MHz}, \mathrm{CD}_{2} \mathrm{Cl}_{2}, 25^{\circ} \mathrm{C}\right) \delta(\mathrm{ppm}) 151.20,147.70,146.77,140.84$, $140.04,138.98,136.47,133.37,132.35,131.93,129.76,129.22,128.29,125.57,125.35,124.70$, 123.99, 122.21, 34.36, 31.09, 20.56; HRMS (ASAP) $(\mathrm{m} / \mathrm{z})$ calculated for $\mathrm{C}_{52} \mathrm{H}_{47} \mathrm{~S}_{2}(\mathrm{M}+\mathrm{H})^{+}$ 735.3119, found 735.3119 .

DihydroIIDBT 2cH2. Following the procedure described above for 2a, dione 5 ( $0.1 \mathrm{~g}, 0.225$ mmol, 1 equiv.) and 1-bromo-4-tert-butylbenzene (0.39 mL, $2.25 \mathrm{mmol}, 10$ equiv.), and $n$-BuLi (1.6 $\mathrm{M}$ in hexanes, $1.34 \mathrm{~mL}, 2.14 \mathrm{mmol}, 9.5$ equiv.) were reacted to yield the crude diol. The diol (0.1 g, $0.14 \mathrm{mmol}, 1$ equiv.) and $\mathrm{SnCl}_{2}\left(0.107 \mathrm{~g}, 0.56 \mathrm{mmol}, 4\right.$ equiv.) were reacted at $70{ }^{\circ} \mathrm{C}$. After $4 \mathrm{~h}$, the mixture was poured over a silica plug and washed with hexanes. Switching to 3:1 hexanes $/ \mathrm{CH}_{2} \mathrm{Cl}_{2}$ eluted the crude product. The dihydro product was further purified using preparative TLC using 2:1 hexanes/ $\mathrm{CH}_{2} \mathrm{Cl}_{2}$ as eluent to furnish $\mathbf{2} \mathbf{c H}_{2}(21 \mathrm{mg}, 23 \%$ from $\mathbf{5})$ as a pale yellow solid. ${ }^{1} \mathrm{H}$ NMR $\left(500 \mathrm{MHz}, \mathrm{CD}_{2} \mathrm{Cl}_{2}, 25^{\circ} \mathrm{C}\right) \delta(\mathrm{ppm}) 8.29(\mathrm{~d}, J=7.9 \mathrm{~Hz}, 2 \mathrm{H}), 8.15(\mathrm{~s}$, 2H), $7.90(\mathrm{~d}, J=7.9 \mathrm{~Hz}, 2 \mathrm{H}), 7.82(\mathrm{~s}, 2 \mathrm{H}), 7.55(\mathrm{t}, J=7.6 \mathrm{~Hz}, 2 \mathrm{H}), 7.41(\mathrm{t}, J=7.6 \mathrm{~Hz}, 2 \mathrm{H}), 7.36$ $(\mathrm{d}, J=7.9 \mathrm{~Hz}, 4 \mathrm{H}), 7.17$ (d, $J=7.9 \mathrm{~Hz}, 4 \mathrm{H}), 5.36$ (s, 2H), 1.31 (s, 18H); ${ }^{13} \mathrm{C} \mathrm{NMR}(126 \mathrm{MHz}$, $\left.\mathrm{CD}_{2} \mathrm{Cl}_{2}, 25^{\circ} \mathrm{C}\right) \delta(\mathrm{ppm}) 152.32,150.37,149.92,145.22,139.70,137.68,136.40,132.90,131.54$, $127.47,125.86,124.98,124.32,123.85,123.68,122.24,116.91,53.85,53.63,53.41,53.20,52.98$, 51.91, 34.40, 31.07, 29.68; HRMS (ASAP) $(\mathrm{m} / z)$ calculated for $\mathrm{C}_{48} \mathrm{H}_{40} \mathrm{~S}_{2}(\mathrm{M}+\mathrm{H})^{+} 680.2572$, found 680.2539 .

anti-IIDBT 1b. Following the procedure described above for $\mathbf{2 a}$, anti-dione ${ }^{8}(0.140 \mathrm{~g}, 0.32$ mmol, 1 equiv.) and 2-bromo-5-t-butyl-1,3-dimethylbenzene (0.608 g, 2.52 mmol, 8 equiv.), and $n$-BuLi (1.6 M in hexanes, $1.50 \mathrm{~mL}, 2.36 \mathrm{mmol}, 7.5$ equiv.) were reacted to give the crude diol. Dearomatization of the crude diol $(0.201 \mathrm{~g}, 0.261 \mathrm{mmol}, 1$ equiv. $)$ with anhydrous $\mathrm{SnCl}_{2}(0.198 \mathrm{~g}$, $1.05 \mathrm{mmol}, 4$ equiv.) gave a deep green solution which was reacted for $4 \mathrm{~h}$ and then filtered through 
a pad of celite. The filtrate was concentrated, triturated with $\mathrm{MeCN}$, and filtered to yield syn-IIDBT $1 \mathbf{b}\left(0.074 \mathrm{~g}, 42 \%\right.$ from anti-dione) as a deep green solid. ${ }^{1} \mathrm{H} \mathrm{NMR}\left(500 \mathrm{MHz}, \mathrm{CD}_{2} \mathrm{Cl}_{2}, 25{ }^{\circ} \mathrm{C}\right) \delta$ (ppm) 7.61 (d, $J=7.7 \mathrm{~Hz}, 2 \mathrm{H}), 7.24-7.13$ (m, overlapping singlets and a triplet, 8H), 7.08 (s, 2H), $6.92(\mathrm{~d}, J=7.8 \mathrm{~Hz}, 2 \mathrm{H}), 6.86(\mathrm{~s}, 2 \mathrm{H}), 2.23(\mathrm{~s}, 12 \mathrm{H}), 1.41(\mathrm{~s}, 18 \mathrm{H}) ;{ }^{13} \mathrm{C} \mathrm{NMR}\left(126 \mathrm{MHz}, \mathrm{CD}_{2} \mathrm{Cl}_{2}\right.$, $\left.25^{\circ} \mathrm{C}\right) \delta(\mathrm{ppm}) 150.94,145.30,144.75,142.54,138.76,138.25,136.66,133.00,131.87,130.93$, $130.59,130.18,127.59,125.54,125.21,124.64,123.88,122.07,34.65,31.62,20.96$; HRMS (ASAP) $(m / z)$ calculated for $\mathrm{C}_{52} \mathrm{H}_{47} \mathrm{~S}_{2}(\mathrm{M}+\mathrm{H})^{+} 735.3119$, found 735.3076 .

Variable Temperature NMR Experiments. Approximately $10 \mathrm{mg}$ of IIDBT 2a was dissolved in 1,2-dichlorobenzene- $d_{4}$ and transferred to an NMR tube. Proton NMR spectra were acquired in a Varian Inova $500 \mathrm{MHz}$ spectrometer that was heated to 50, 75, 100, 130, 140, then cooled back to $25^{\circ} \mathrm{C}$.

General Details for X-ray Structures. Diffraction intensities for 1d were collected at 173 $\mathrm{K}$ on a Bruker Apex $2 \mathrm{CCD}$ diffractometer using $\mathrm{CuK} \alpha$ radiation, $\lambda=1.54178 \AA$. Space group was determined based on intensity statistics. Absorption correction was applied by SADABS. ${ }^{11}$ Structure was solved by direct methods and Fourier techniques and refined on $F^{2}$ using full matrix least-squares procedures. All non-H atoms were refined with anisotropic thermal parameters. H atoms in all structures were refined in calculated positions in a rigid group model. Solvent molecule, $\mathrm{CH}_{3} \mathrm{CN}$, is highly disordered over three or four positions. Some of these positions are related by an inversion center. This solvent molecule was treated by SQUEEZE. ${ }^{12}$ The correction of the X-ray data by SQUEEZE was 51 electron/cell; the expected number of electrons is 44 electrons/cell. Crystals of $\mathbf{1 d}$ were very thin plates that did not provide visible reflections at high angles. Even using a strong Incoatec I $\mu S \mathrm{Cu}$ source it was possible to collect diffraction data only up to $2 \theta_{\max }=101.25^{\circ}$. The collected data provide 2737 reflections per 271 refined parameters. All calculations were performed by the Bruker SHELXL-2014 package. ${ }^{13}$

X-ray diffraction data for $\mathbf{2 b}$ were collected on a Rigaku XtaLAB Synergy diffractometer coupled to a Rigaku Hypix detector with $\mathrm{Cu} \operatorname{K} \alpha$ radiation $(\lambda=1.54184 \AA)$, from a PhotonJet microfocus X-ray source at $200 \mathrm{~K}$. The diffraction images were processed and scaled using the CrysAlisPro software. ${ }^{14}$ The structures were solved through intrinsic phasing using SHELXT ${ }^{13}$ and refined against $\mathrm{F}^{2}$ on all data by full-matrix least squares with SHELXL $^{15}$ following established refinement strategies. ${ }^{16}$ All non-hydrogen atoms were refined anisotropically. All hydrogen atoms bound to carbon were included in the model at geometrically calculated positions 
and refined using a riding model. The isotropic displacement parameters of all hydrogen atoms were fixed to 1.2 times the Ueq value of the atoms they are linked to (1.5 times for Me groups).

Crystallographic Data for 1d. $\mathrm{C}_{62} \mathrm{H}_{64} \mathrm{~N}_{2} \mathrm{~S}_{2}, \mathrm{C}_{58} \mathrm{H}_{58} \mathrm{~S}_{2} \cdot 2\left(\mathrm{CH}_{3} \mathrm{CN}\right), \mathrm{M}=901.27,0.12 \times 0.09 \times$ $0.01 \mathrm{~mm}, \mathrm{~T}=173(2) \mathrm{K}$, Triclinic, space group $P-1, a=9.0360(6) \AA, b=10.7067(7) \AA, c=$ 14.4033(10) $\AA, \alpha=77.940(4)^{\circ}, \beta=85.741(5)^{\circ}, \gamma=74.769(4)^{\circ}, V=1314.56(16) \AA^{3}, Z=1, D_{\mathrm{c}}=$ $1.138 \mathrm{Mg} / \mathrm{m}^{3}, \mu(\mathrm{Cu})=1.210 \mathrm{~mm}^{-1}, F(000)=482,2 \theta_{\max }=101.25^{\circ}, 9539$ reflections, 2737 independent reflections $\left[\mathrm{R}_{\mathrm{int}}=0.0436\right], \mathrm{R} 1=0.0545, \mathrm{wR} 2=0.1503$ and $\mathrm{GOF}=1.068$ for 2737 reflections (271 parameters) with $\mathrm{I}>2 \sigma(\mathrm{I}), \mathrm{R} 1=0.0755, \mathrm{wR} 2=0.1593$ and $\mathrm{GOF}=1.068$ for all reflections, $\max / \mathrm{min}$ residual electron density $+0.337 /-0.166 \mathrm{e}^{-3}$. CCDC 1589136.

Crystallographic Data for $\mathbf{2} \mathbf{b} . \mathrm{C}_{54} \mathrm{H}_{48} \mathrm{Cl}_{6} \mathrm{~S}_{2}, \mathrm{C}_{52} \mathrm{H}_{46} \mathrm{~S}_{2} \cdot 2\left(\mathrm{CHCl}_{3}\right), \mathrm{M}=973.74,0.185 \times 0.073$ x $0.037 \mathrm{~mm}^{3}, \mathrm{~T}=200.00(10) \mathrm{K}$, Monoclinic, space group P2 $1 / \mathrm{c}, a=8.11560(10) \AA, b=$ 9.97380(10) $\AA, c=29.2458(3) \AA, \alpha=90^{\circ}, \beta=94.8550(10)^{\circ}, \gamma=90^{\circ}, V=2358.76(4) \AA^{3}, Z=2$, $D_{c}=1.371 \mathrm{Mg} / \mathrm{m}^{3}, \mu(\mathrm{Cu})=4.432 \mathrm{~mm}^{-1}, F(000)=1012,2 \theta_{\max }=67.723^{\circ}, 29112$ reflections, 4271 independent reflections $[\mathrm{R}(\mathrm{int})=0.0397], \mathrm{R} 1=0.0847, \mathrm{wR} 2=0.2315$ and $\mathrm{GOF}=1.041$ for all reflections, $\max / \mathrm{min}$ residual electron density $+1.232 /-0.929 \mathrm{e}^{-3}$. CCDC 1949404.
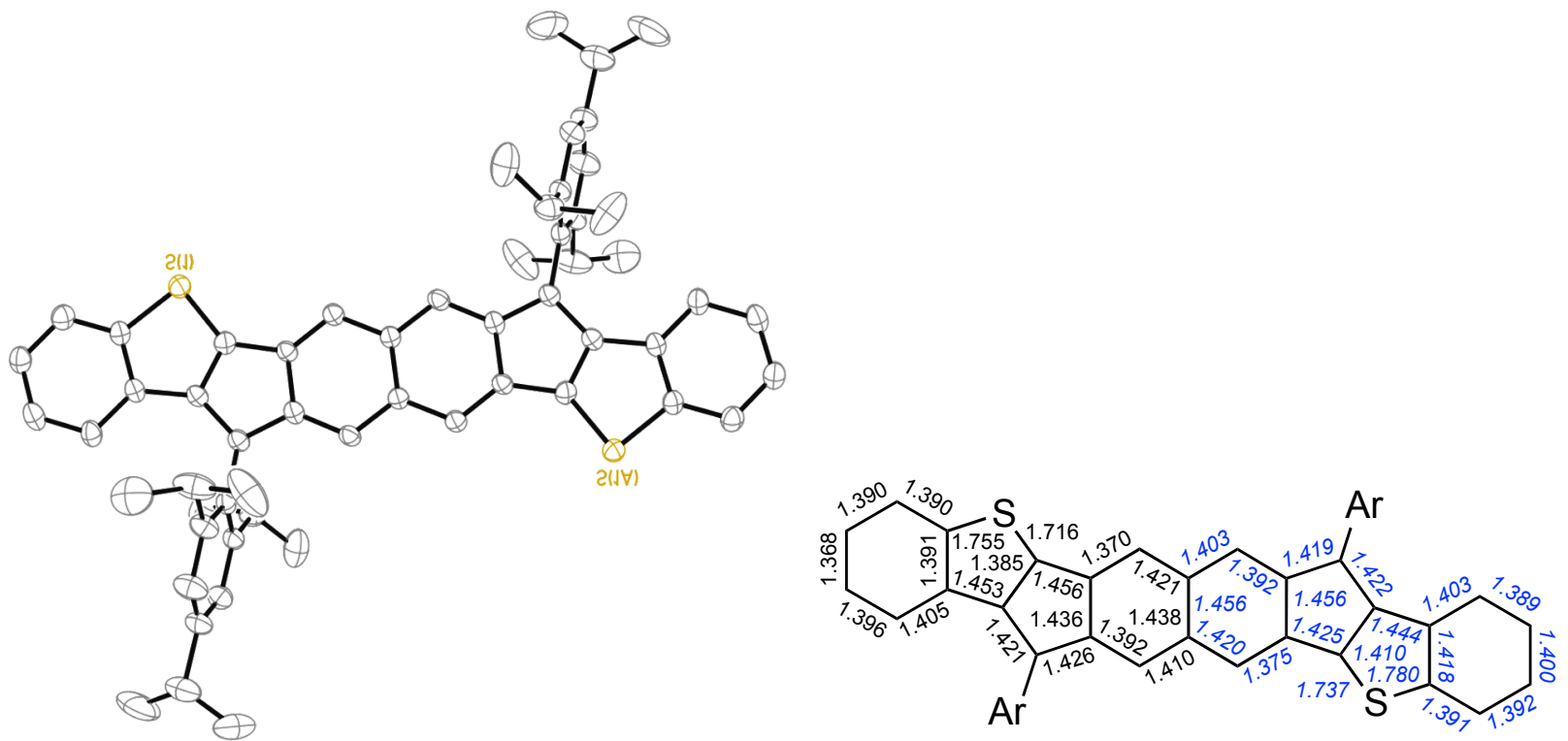

Figure S5. (left) Molecular structure of $\mathbf{1 d}$ with the ellipsoids drawn with 50\% probability level; hydrogens are omitted for clarity. (right) Experimental (black) bond lengths for the core motif of 1d along with the calculated (blue) values for $1 \mathbf{a}$. 
TGA measurements. Thermogravimetric Analysis (TGA) was performed from $25-1000{ }^{\circ} \mathrm{C}$ on samples of 1a-1b and 2a-2b using a TGA Q500 V6.7 Build 203 instrument. The measurements were performed under an oxygen-free dry $\mathrm{N}_{2}$ flow.

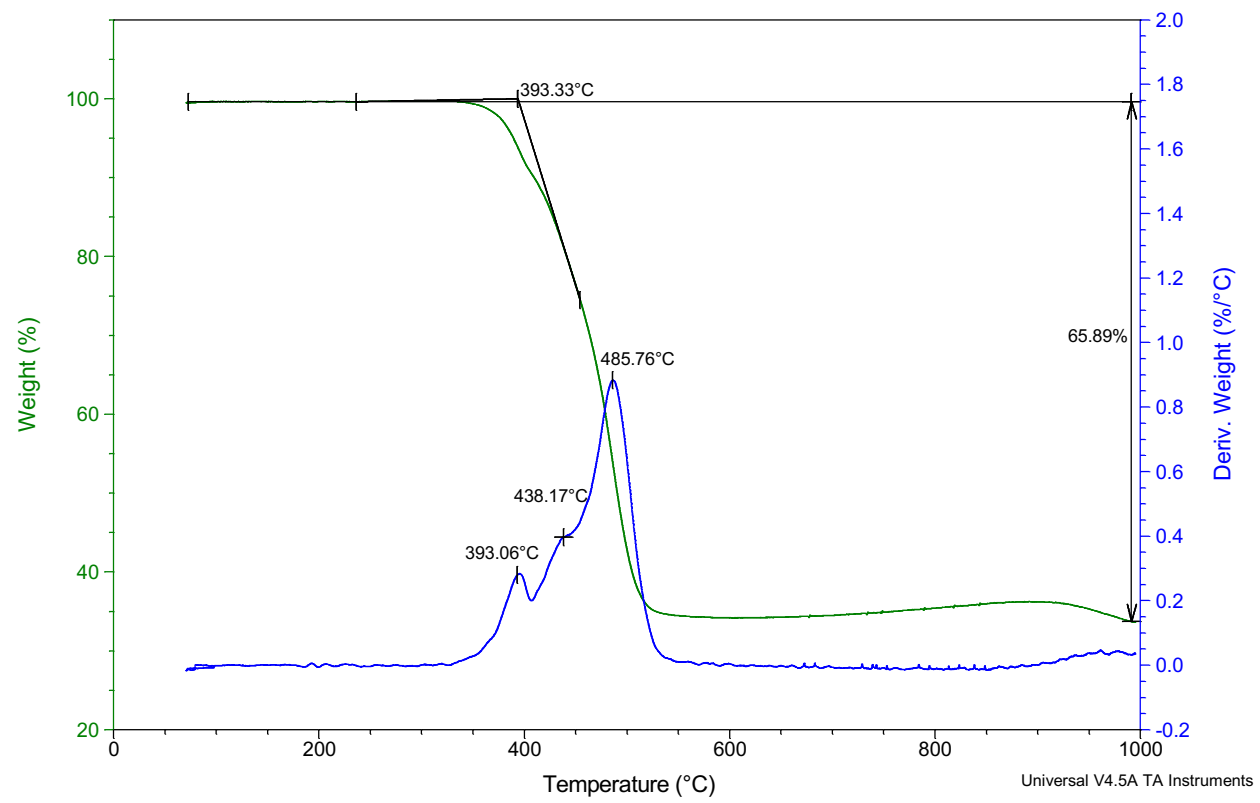

Figure S6. TGA plot for anti-IIDBT 1a.

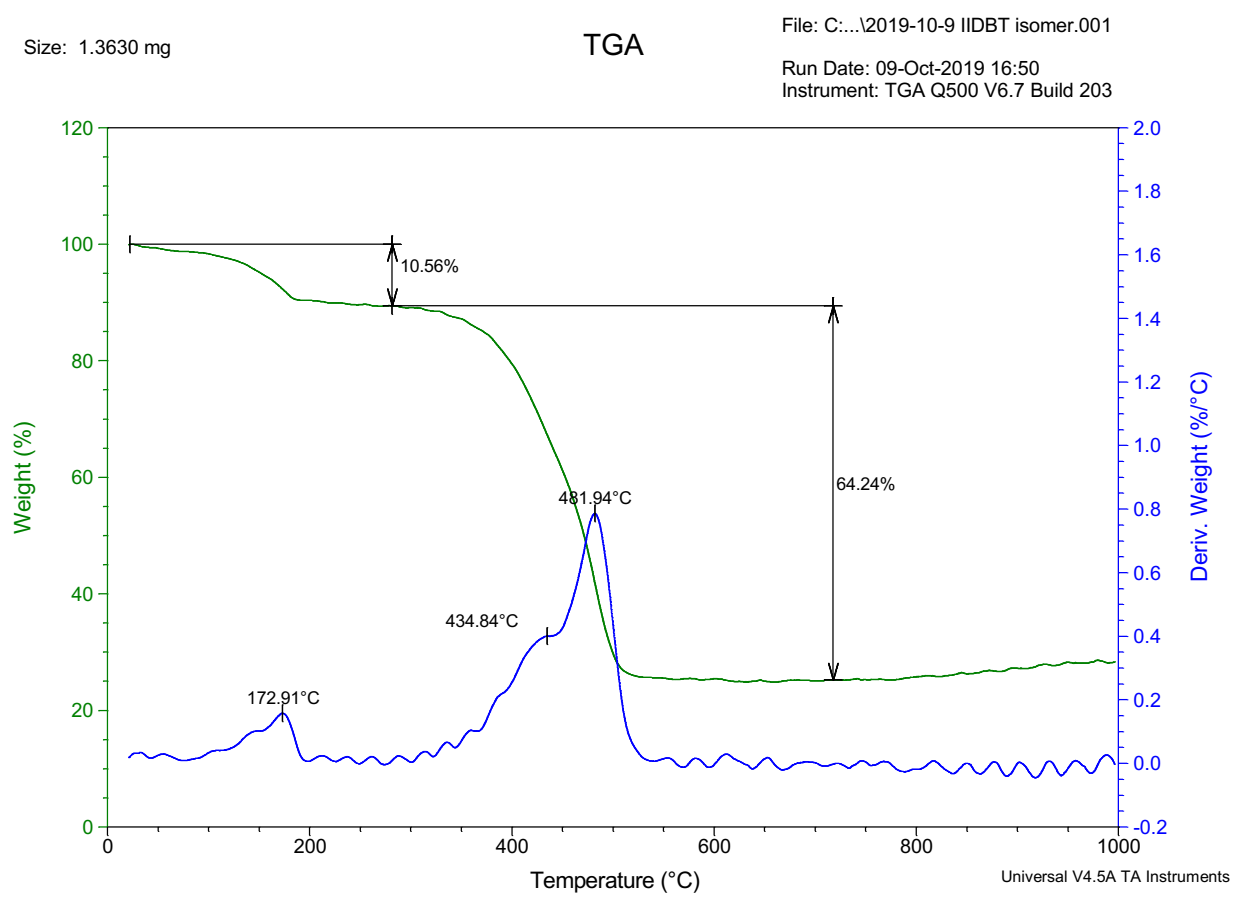

Figure S7. TGA plot for anti-IIDBT 1b. The small, initial weight loss corresponds to 1,2-dichlorobenzene solvent. 


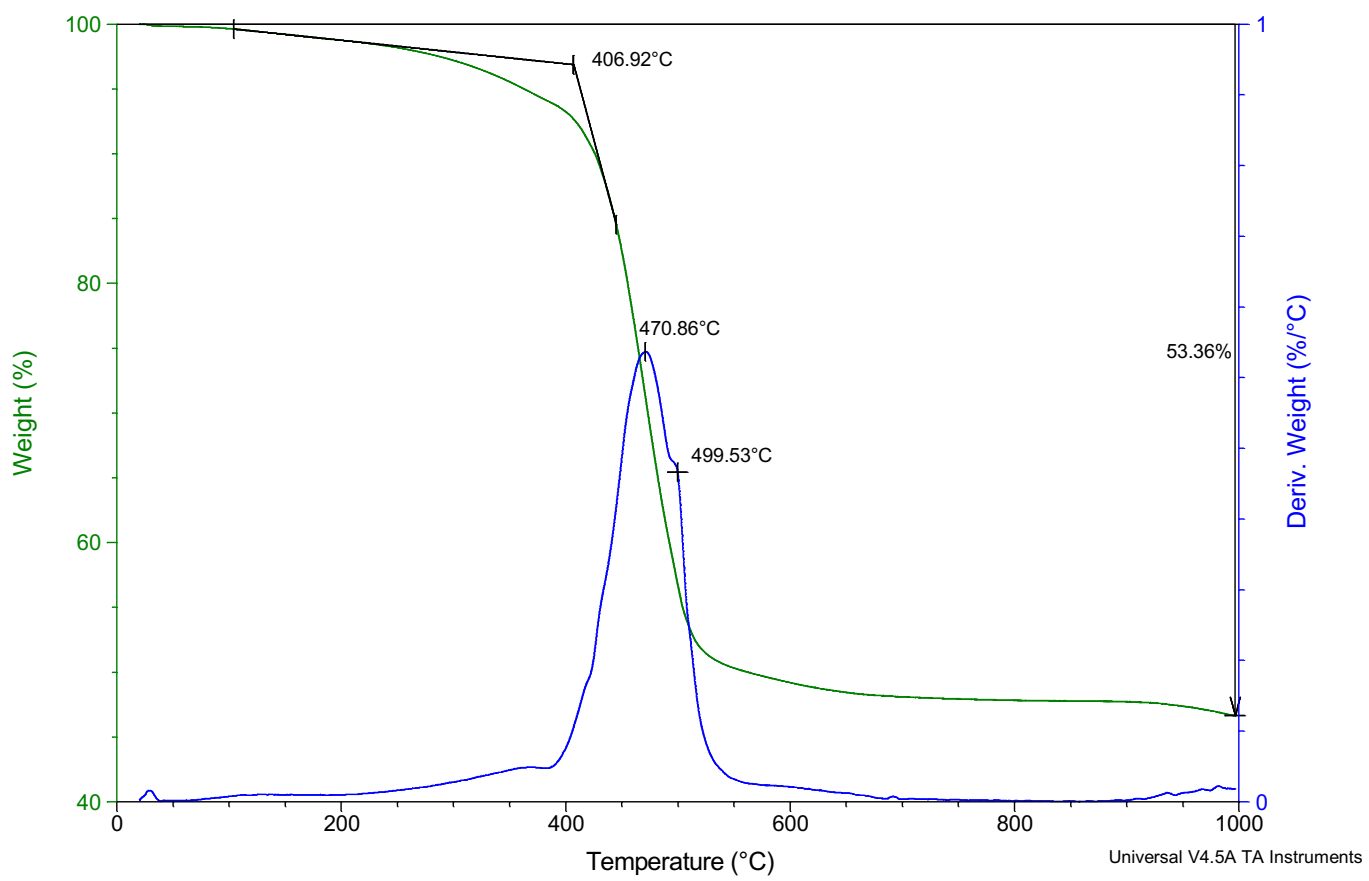

Figure S8. TGA plot for $s y n$-IIDBT $2 \mathbf{a}$.

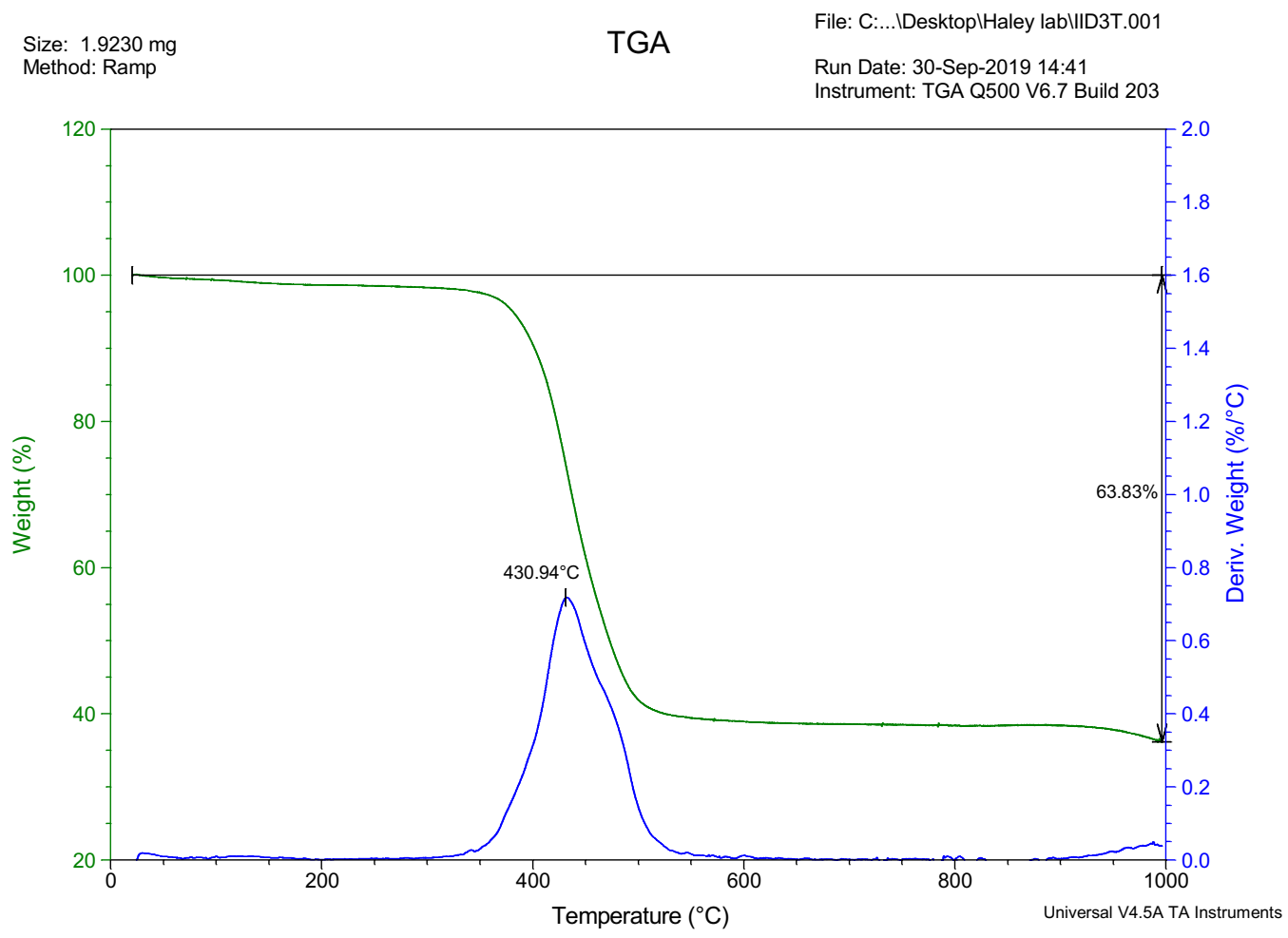

Figure S9. TGA plot for syn-IIDBT $\mathbf{2 b}$. 
SQUID measurements. Magnetic susceptibility measurements were performed with a Quantum Design MPMS-XL-7 SQUID susceptometer equipped with a sample space oven reaching a maximum temperature of $800 \mathrm{~K}$. The magnetic measurements were performed in the 300-700 K temperature range with an applied field of $1 \mathrm{~T}$ using a sample space oven with the samples inserted in a $1.5 \mathrm{~mm}$ diameter aluminium foil cylinder. The sample masses used for the magnetic measurements are 11.665, 3.868 and $1.469 \mathrm{mg}$ for $\mathbf{2 a}, \mathbf{1 b}$ and $\mathbf{2 b}$, respectively. The susceptibility data were corrected for the same sample holder previously measured using the same conditions and for the diamagnetic contributions of the compound as deduced by using Pascal's constant tables. ${ }^{17}$ The magnetic measurements were fitted using the classical Bleaney-Bowers model for an antiferromagentic $\mathrm{S}=1 / 2$ dimer. ${ }^{18}$ This model reproduces satisfactorily the magnetic properties of $2 \mathbf{a}$ with $\mathrm{g}=2.0(1), \mathrm{J}=-3479 \mathrm{~K}$ and a paramagnetic $\mathrm{S}=1 / 2$ impurity $(\rho)$ of $c a .0 .6 \%$ with a regression factor, $\mathrm{R}=0.99877$ (the Hamiltonian is written as $\mathrm{H}=-\mathrm{JS}_{1} \mathrm{~S}_{2}$ ). This $\mathrm{J}$ value corresponds to a $\Delta E_{\mathrm{ST}}$ of $c a .-6.9 \mathrm{kcal} \mathrm{mol}^{-1}$.

Compounds $\mathbf{1 b}$ and $\mathbf{2 b}$ were measured in the heating and cooling scans in order to verify stability. The magnetic measurements of $\mathbf{1 b}$ and $\mathbf{2} \mathbf{b}$ were also fitted to the classical BleaneyBowers model. ${ }^{18}$ This model for $1 \mathbf{b}$ gives (solid lines in Figure S8): $g=2.0(1), \mathrm{J}=-4141 \mathrm{~K}$ and $\rho$ $\approx 0.1 \%$ in the heating $\operatorname{scan}(\mathrm{R}=0.9991)$ and $\mathrm{g}=2.0(1), \mathrm{J}=-4227 \mathrm{~K}$ and $\rho \approx 0.1 \%$ in the cooling scan $(\mathrm{R}=0.9985)$. These $\mathrm{J}$ values correspond to $\Delta E_{\mathrm{ST}}$ of $c a .-8.2$ and $-8.4 \mathrm{kcal} \mathrm{mol}^{-1}$, for the heating and cooling scans, respectively. For $\mathbf{2 b}$ we obtain (solid lines in Figure S9): $g=2.0(1), \mathrm{J}$ $=-3652 \mathrm{~K}$ and $\rho \approx 0.1 \%$ in the heating $\operatorname{scan}(\mathrm{R}=0.9994)$ and $\mathrm{g}=2.0(1), \mathrm{J}=-3533 \mathrm{~K}$ and $\rho \approx$ $0.2 \%$ in the cooling scan $(\mathrm{R}=0.9979)$. These $\mathrm{J}$ values correspond to $\Delta E_{\mathrm{ST}}$ of $c a .-7.2$ and $-7.0 \mathrm{kcal}$ $\mathrm{mol}^{-1}$, for the heating and cooling scans, respectively.

In all cases the paramagnetic impurities may come from a small fraction of mono-radical present in the sample. Given the low magnetic signal, the J value obtained presents an uncertainty of around $10 \%$, as this error corresponds to the error in the $\chi_{\mathrm{m}} \mathrm{T}$ values (and, therefore in the $\mathrm{g}$ values that are directly related to them). For the estimated $\Delta \mathrm{E}_{\mathrm{ST}}$ values from the fit to the BleaneyBowers model, however, the $\Delta \mathrm{E}_{\mathrm{ST}}$ value mainly depends on the curvature of the plot as $\mathrm{T}$ increases; thus, the error in $\Delta \mathrm{E}_{\mathrm{ST}}$ can be evaluated to be around $0.2 \mathrm{kcal} \mathrm{mol}^{-1}$, which is more like $2-3 \%$. 


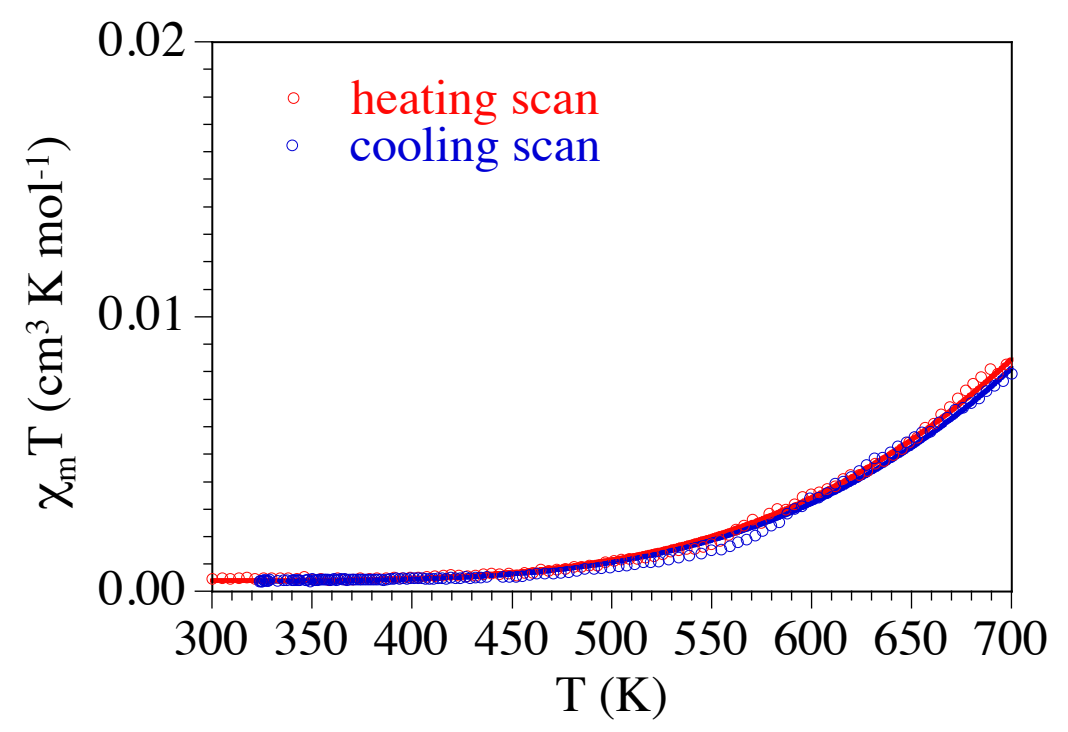

Figure S10. Variable temperature SQUID measurement of anti-IIDBT $\mathbf{1 b}$ in the heating (red points) and cooling scans (blue points). Solid lines are the best fit to the Bleaney-Bowers dimer model.

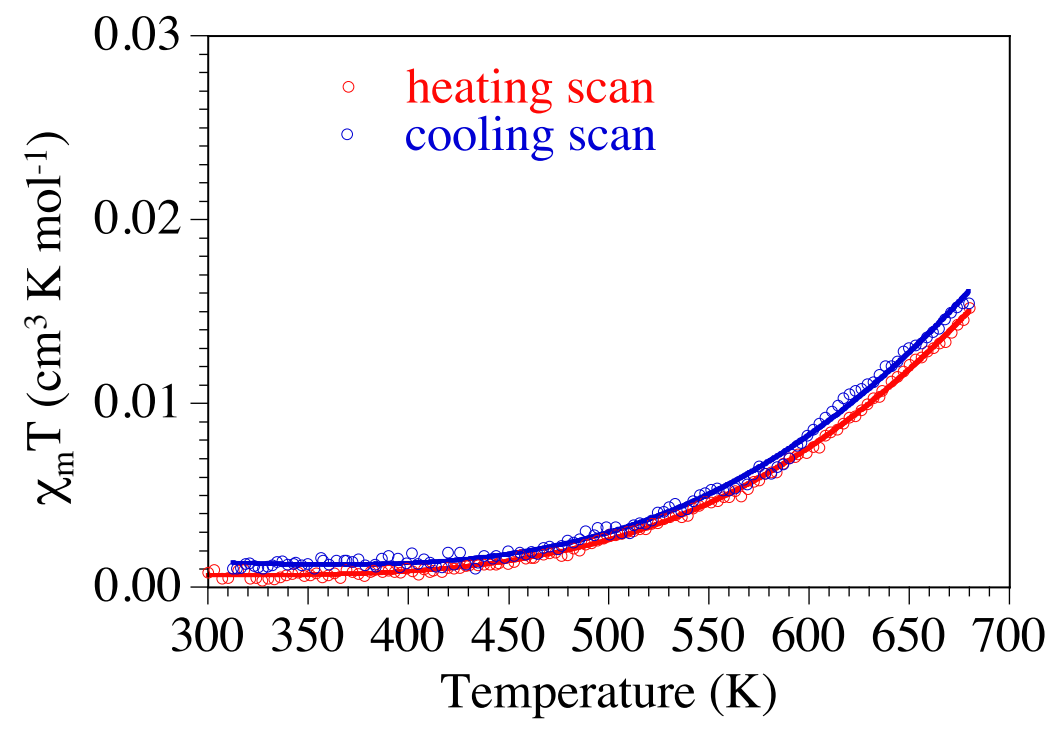

Figure S11. Variable temperature SQUID measurement of syn-IIDBT $\mathbf{2 b}$ in the heating (red points) and cooling scans (blue points). Solid lines are the best fit to the Bleaney-Bowers dimer model. 


\section{Copies of NMR Spectra}

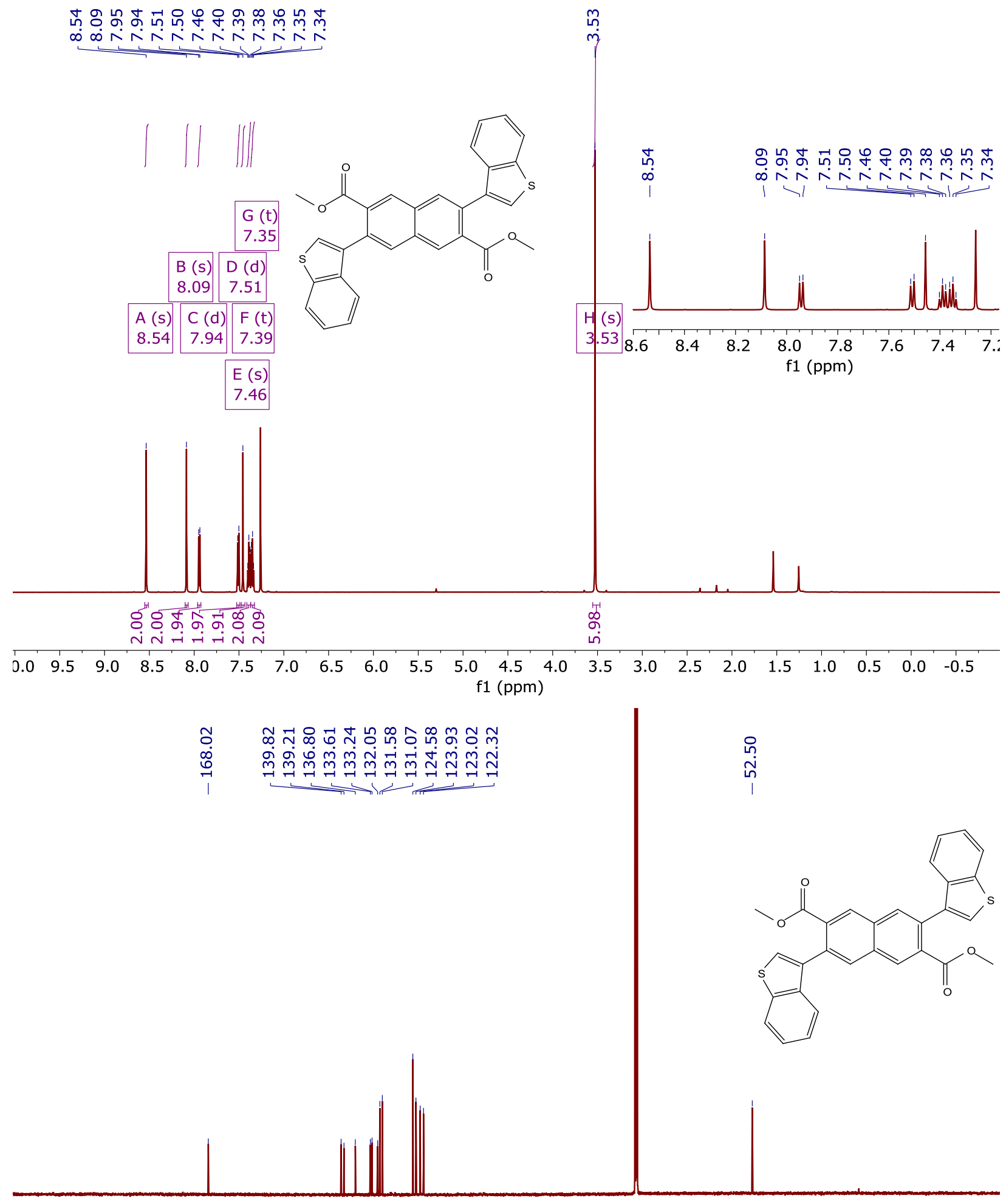

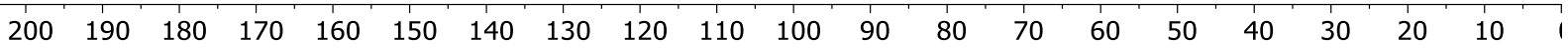
$\mathrm{f1}(\mathrm{ppm})$ 


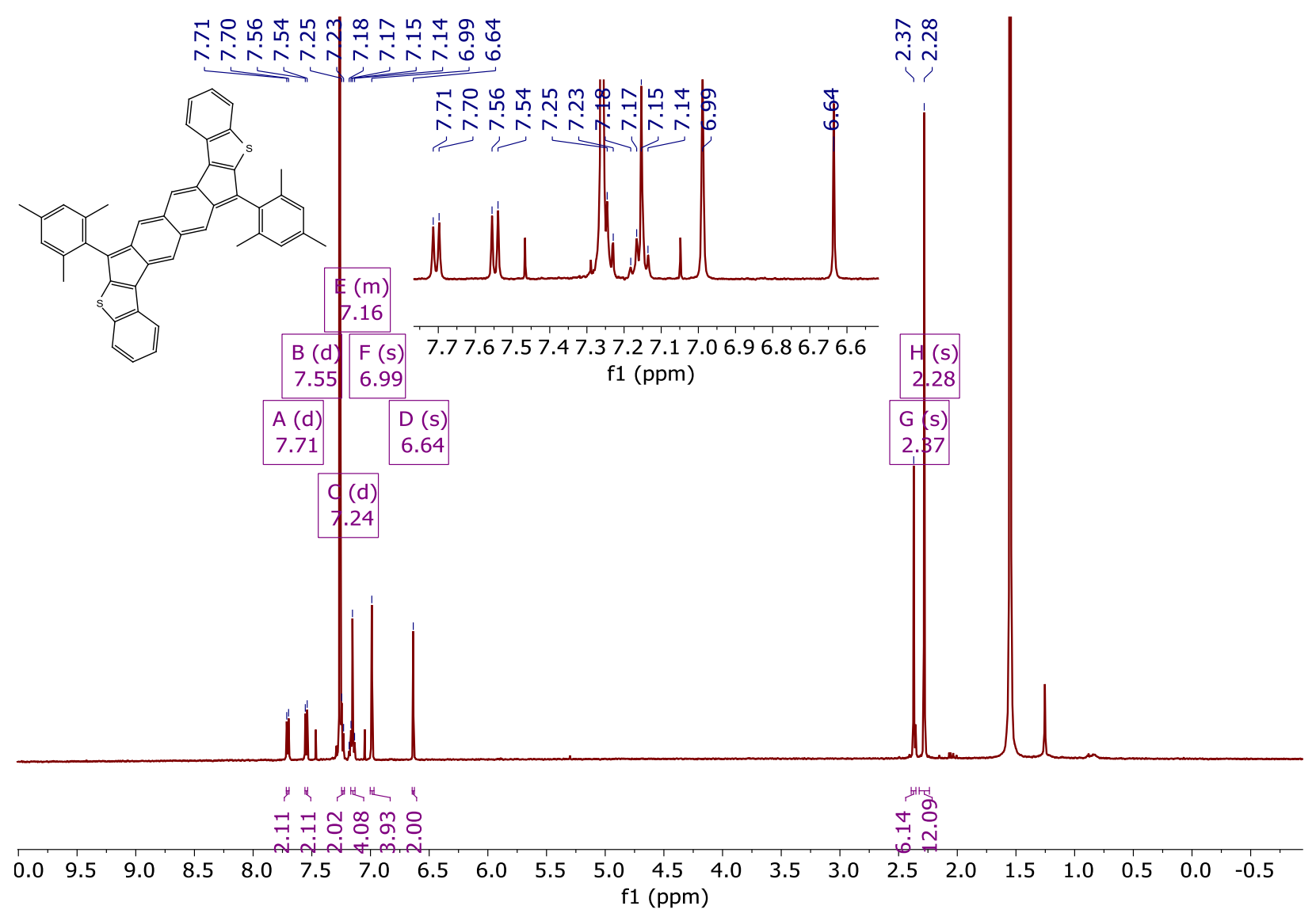




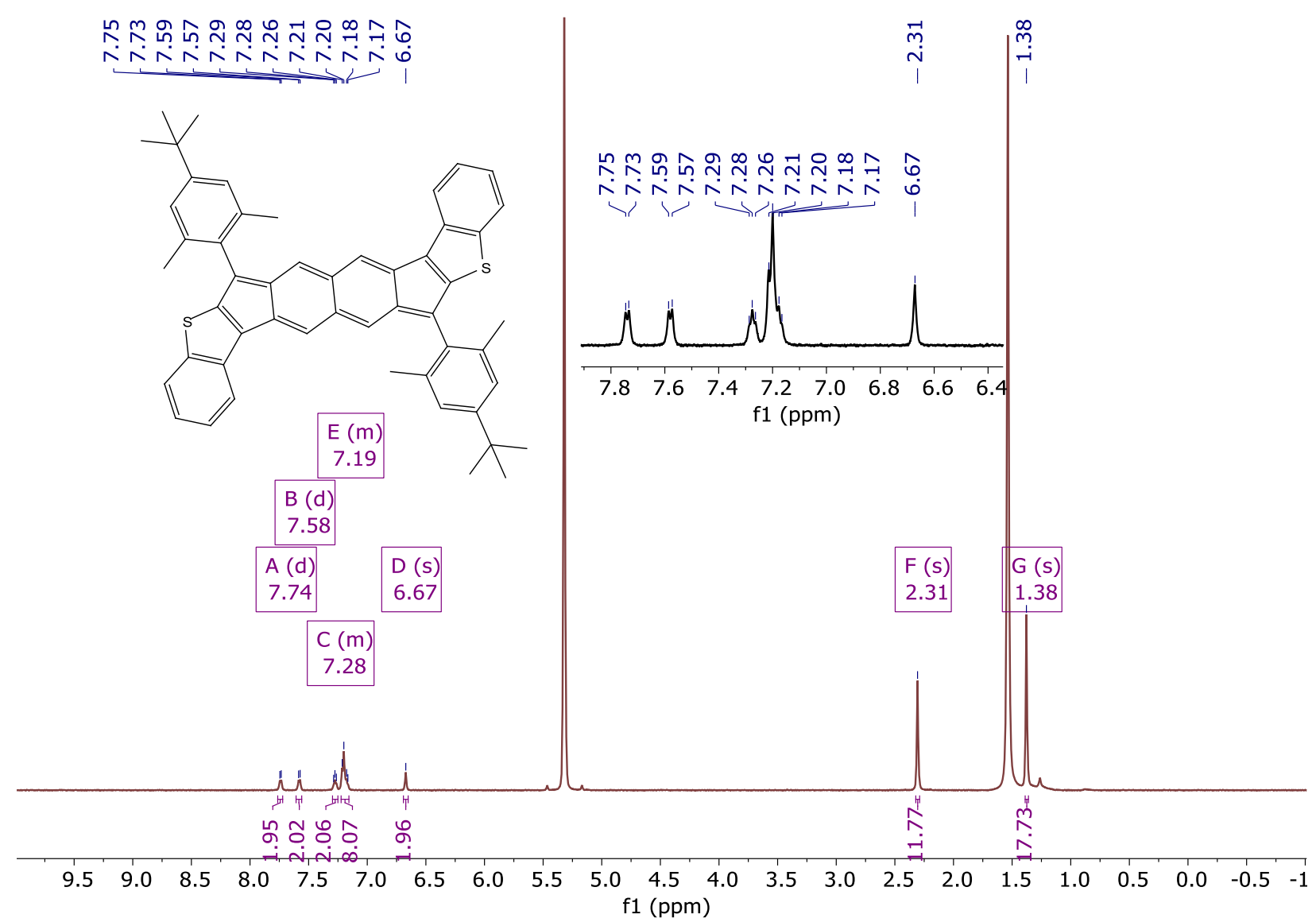

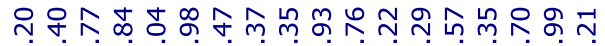

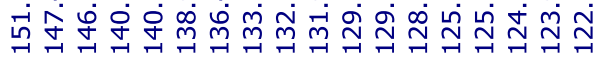

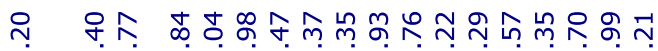

点 $\quad$ 守守

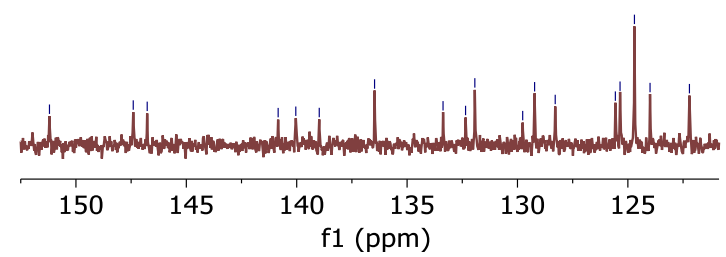

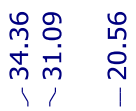

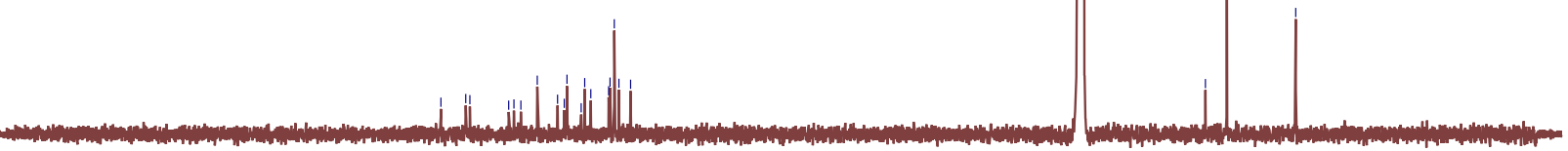

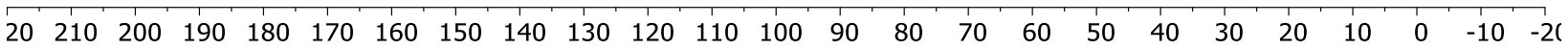
f1 (ppm) 


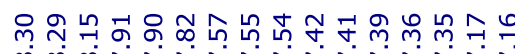

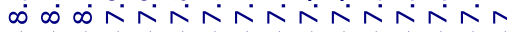

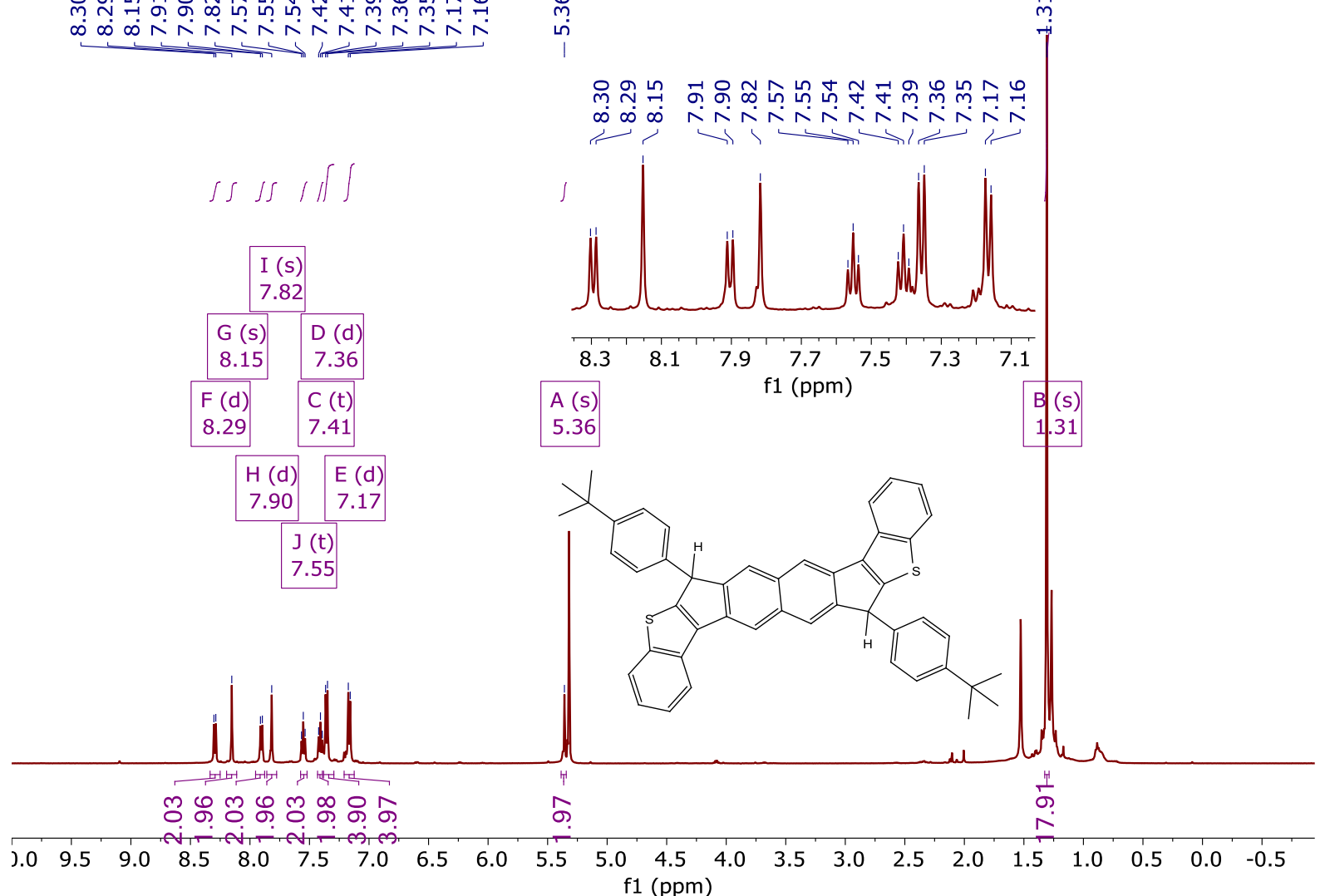

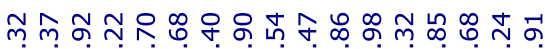

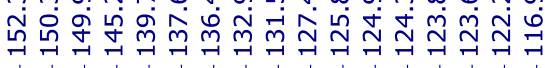

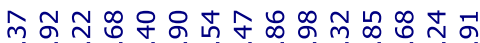

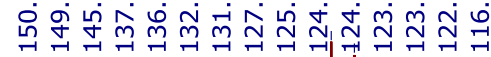
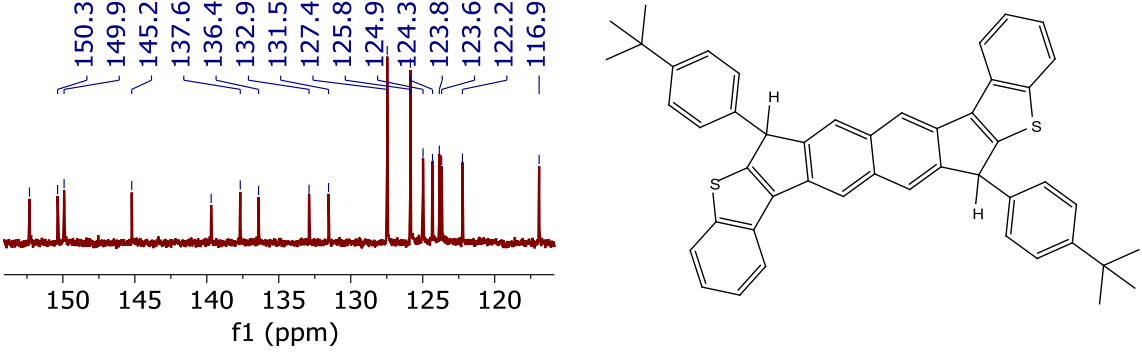

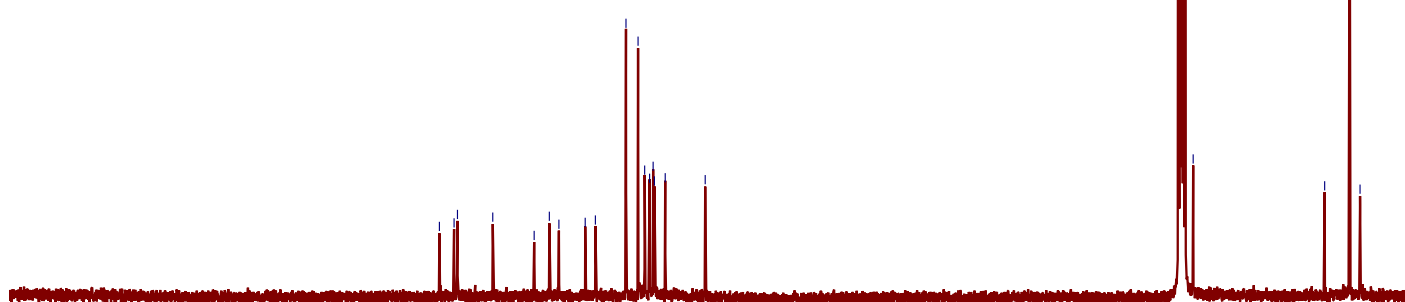




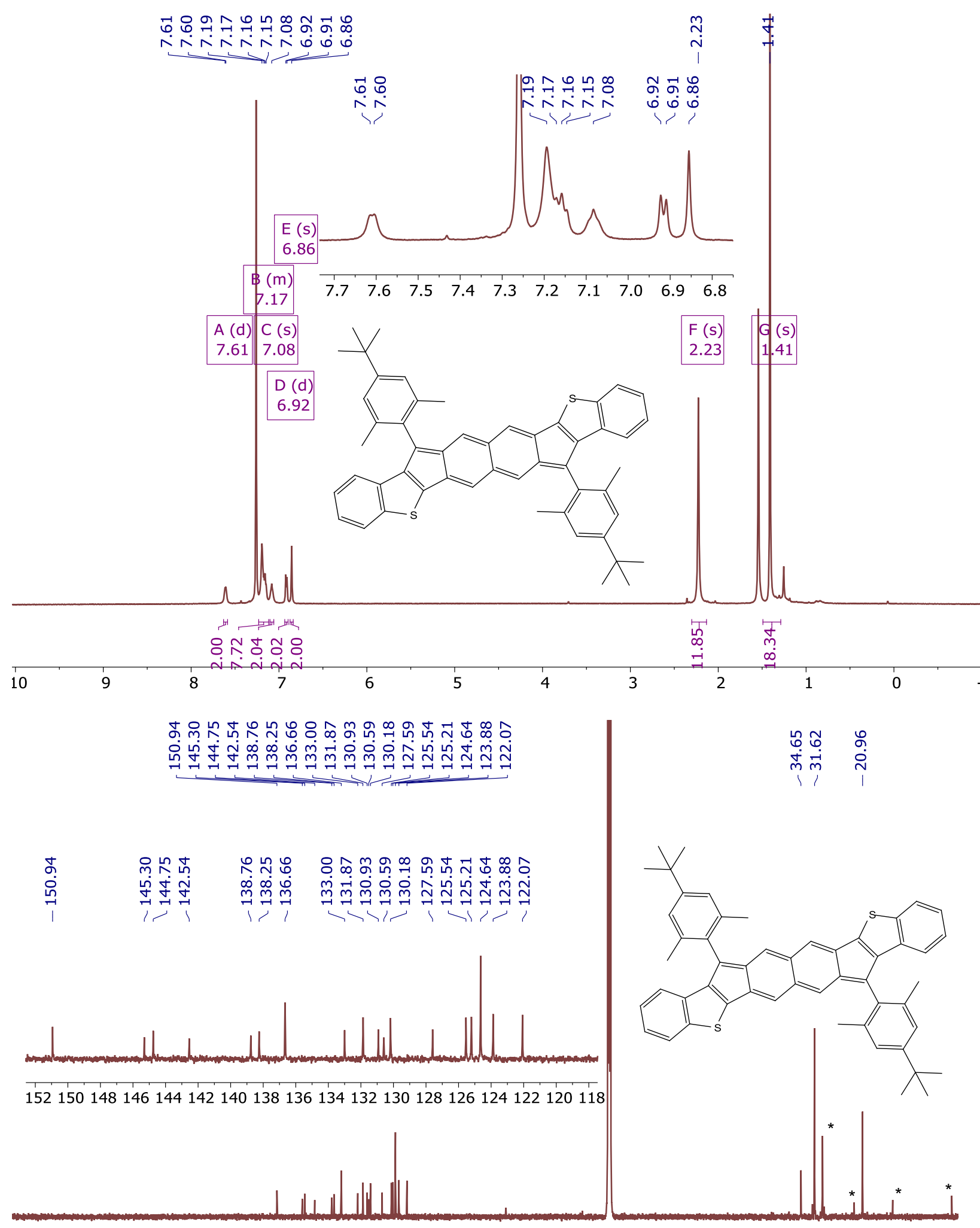

$\begin{array}{lllllllllllllllllllll}200 & 190 & 180 & 170 & 160 & 150 & 140 & 130 & 120 & 110 & 100 & 90 & 80 & 70 & 60 & 50 & 40 & 30 & 20 & 10 & 0\end{array}$ 


\section{References}

(1) Shao, Y.; Head-Gordon, M.; Krylov, A. I. J. Chem. Phys. 2003, 118, 4807.

(2) (a) Wang, F.; Ziegler, T. J. Chem. Phys. 2004, 121, 12191; (b) Wang, F.; Ziegler, T. J. Chem. Phys. 2005, 122, 074109. c) Wang, F.; Ziegler, T. Int. J. Quantum Chem. 2006, 106, 2545.

(3) Stein, T.; Eisenberg, H.; Kronik, L.; Baer, R. Phys. Rev. Lett. 2010, 105, 266802.

(4) Frisch, M. J.; Trucks, G. W.; Schlegel, H. B.; Scuseria, G. E.; Robb, M. A.; Cheeseman, J. R.; Scalmani, G.; Barone, V.; Mennucci, B.; Petersson, G. A.; Nakatsuji, H.; Caricato, M.; Li, X.; Hratchian, H. P.; Izmaylov, A. F.; Bloino, J.; Zheng, G.; Sonnenberg, J. L.; Hada, M.; Ehara, M.; Toyota, K.; Fukuda, R.; Hasegawa, J.; Ishida, M.; Nakajima, T.; Honda, Y.; Kitao, O.; Nakai, H.; Vreven, T.; Montgomery, J. A., J.; Peralta, J. E.; Ogliaro, F.; Bearpark, M.; Heyd, J. J.; Brothers, E.; Kudin, K. N.; Staroverov, V. N.; Kobayashi, R.; Normand, J.; Raghavachari, K.; Rendell, A.; Burant, J. C.; Iyengar, S. S.; Tomasi, J.; Cossi, M.; Rega, N.; Millam, J. M.; Klene, M.; Knox, J. E.; Cross, J. B.; Bakken, V.; Adamo, C.; Jaramillo, J.; Gomperts, R.; Stratmann, R. E.; Yazyev, O.; Austin, A. J.; Cammi, R.; Pomelli, C.; Ochterski, J. W.; Martin, R. L.; Morokuma, K.; Zakrzewski, V. G.; Voth, G. A.; Salvador, P.; Dannenberg, J. J.; Dapprich, S.; Daniels, A. D.; Farkas, Ö.; Foresman, J. B.; Ortiz, J. V.; Cioslowski, J.; Fox, D. J. Gaussian 09; Revision D.01; Gaussian, Inc.: Wallingford CT, 2009.

(5) Schmidt, M. W.; Baldridge, K. K.; Boatz, J. A.; Elbert, S. T.; Gordon, M. S.; Jensen, J. H.; Koseki, S.; Matsunaga, N.; Nguyen, K. A.; Su, S.; Windus, T. L.; Dupuis, M.; Montgomery, J. A. J. Comput. Chem. 1993, 14, 1347.

(6) Shao, Y.; Gan, Z.; Epifanovsky, E.; Gilbert, A. T. B.; Wormit, M.; Kussmann, J.; Lange, A. W.; Behn, A.; Deng, J.; Feng, X.; Ghosh, D.; Goldey, M.; Horn, P. R.; Jacobson, L. D.; Kaliman, I.; Khaliullin, R. Z.; Kuś, T.; Landau, A.; Liu, J.; Proynov, E. I.; Rhee, Y. M.; Richard, R. M.; Rohrdanz, M. A.; Steele, R. P.; Sundstrom, E. J.; Woodcock, H. L.; Zimmerman, P. M.; Zuev, D.; Albrecht, B.; Alguire, E.; Austin, B.; Beran, G. J. O.; Bernard, Y. A.; Berquist, E.; Brandhorst, K.; Bravaya, K. B.; Brown, S. T.; Casanova, D.; Chang, C.-M.; Chen, Y.; Chien, S. H.; Closser, K. D.; Crittenden, D. L.; Diedenhofen, M.; DiStasio, R. A.; Do, H.; Dutoi, A. D.; Edgar, R. G.; Fatehi, S.; Fusti-Molnar, L.; Ghysels, A.; Golubeva-Zadorozhnaya, A.; Gomes, J.; Hanson-Heine, M. W. D.; Harbach, P.H.P.; Hauser, A. W. ; Hohenstein, E. G.; Holden, Z. C.; Jagau, T.-C.; Ji, H.; Kaduk, B.n; Khistyaev, K.; Kim, J.; Kim, J.; King, R. A.; Klunzinger, P.; Kosenkov, D.; Kowalczyk, T.; Krauter, C. M.; Lao, K. U.; Laurent, A. D.; Lawler, K. V.; Levchenko, S. V.; Lin, C. Y.; Liu, F.; Livshits, E.; Lochan, R. C.; Luenser, A.; Manohar, P.; Manzer, S. F.; Mao, S.-P.; Mardirossian, N.; Marenich, A. V.; Maurer, S. A.; Mayhall, N. J.; Neuscamman, E.; Oana, C. M.; Olivares- 
Amaya, R.; O’Neill, D. P.; Parkhill, J. A.; Perrine, T. M.; Peverati, R.; Prociuk, A.; Rehn, D. R.; Rosta, E.; Russ, N. J.; Sharada, S. M.; Sharma, S.; Small, D. W.; Sodt, A.; Stein, T.; Stück, D.; Su, Y.-C.; Thom, A. J. W.; Tsuchimochi, T.; Vanovschi, V.; Vogt, L.; Vydrov, O.; Wang, T.; Watson, M. A.; Wenzel, J.; White, A.; Williams, C. F.; Yang, J.; Yeganeh, S.; Yost, S. R.; You, Z.-Q.; Zhang, I. Y.; Zhang, X.; Zhao, Y.; Brooks, B. R.; Chan, G. K. L.; Chipman, D. M.; Cramer, C. J.; Goddard, W. A.; Gordon, M. S.; Hehre, W. J.; Klamt, A.; Schaefer, H. F.; Schmidt, M. W.; Sherrill, C. D.; Truhlar, D. G.; Warshel, A.; Xu, X.; Aspuru-Guzik, A.; Baer, R.; Bell, A. T.; Besley, N. A.; Chai, J.-D.; Dreuw, A.; Dunietz, B. D.; Furlani, T. R.; Gwaltney, S. R.; Hsu, C.-P.; Jung, Y.; Kong, J.; Lambrecht, D. S.; Liang, W.; Ochsenfeld, C.; Rassolov, V. A.; Slipchenko, L.V.; Subotnik, J. E.; Voorhis, T. V.; Herbert, J. M.; Krylov, A. I.; Gill, P. M.W.; Head-Gordon, M. Mol. Phys. 2015, $113,184$.

(7) Nakano, M.; Fukui, H.; Minami, T.; Yoneda, K.; Shigeta, Y.; Kishi, R.; Champagne, B.; Botek, E.; Kubo, T.; Ohta, K. Theor. Chem. Acc. 2011, 130, 711.

(8) Dressler, J. J.; Teraoka, M.; Espejo, G. L.; Kishi, R.; Takamuku, S.; Gómez-García, C. J.; Zakharov, L. N.; Nakano, M.; Casado, J.; Haley, M. M. Nat. Chem. 2018, 10, 1134-1140.

(9) Knall, A.-C.; Ashraf, R. S.; Nikolka, M.; Nielsen, C. B.; Purushothaman, B.; Sadhanala, A.; Hurhangee, M.; Broch, K.; Harkin, D. J.; Novák, J.; Neophytou, M.; Hayoz, P.; Sirringhaus, H.; McCulloch, I. Adv. Funct. Mater. 2016, 26, 6961-6969.

(10) Nakagawa, H.; Kawai, S.; Nakashima, T.; Kawai, T. Org. Lett. 2009, 11, 1475-1478.

(11) Sheldrick, G. M. Bruker/Siemens Area Detector Absorption Correction Program, Bruker AXS, Madison, WI, 1998.

(12) Spek, A. L. Acta Cryst., Sect. C 2015, C71, 9-18.

(13) Sheldrick, G. M. Acta Cryst., Sect. C 2015, C71, 3-8.

(14) CrysAlisPro, Rigaku OD, The Woodlands, TX, 2015.

(15) Sheldrick, G. M. Acta Cryst., Sect. A 2008, A64, 112-122.

(16) Müller, P. Crystallogr. Rev. 2009, 15, 57-83.

(17) Bain, G. A.; Berry, J. F. Diamagnetic corrections and Pascal's constants. J. Chem. Educ. 2008, $85,532-536$.

(18) Bleaney, B.; Bowers, K. D. Anomalous Paramagnetism of Copper Acetate. Proc. R. Soc. Lond. A. 1952, 214, 451-465. 